\title{
Analysis of three-spacecraft data using planar reciprocal vectors: methodological framework and spatial gradient estimation
}

\author{
J. $\operatorname{Vogt}^{1}$, A. Albert $^{1}$, and O. Marghitu ${ }^{2}$ \\ ${ }^{1}$ School of Engineering and Science, Jacobs University Bremen, Campus Ring 1, 28759 Bremen, Germany \\ ${ }^{2}$ Institute for Space Sciences, Bucharest, Romania \\ Received: 31 January 2009 - Revised: 25 June 2009 - Accepted: 6 July 2009 - Published: 19 August 2009
}

\begin{abstract}
In the context of ESA's Cluster mission, four-point array techniques are widely used to analyze space plasma phenomena such as shocks and discontinuities, waves and turbulence, and spatial gradients. Due to failures of single instruments on the Cluster spacecraft fleet, there is also need for array processing of three-point measurements. In this paper we identify planar reciprocal vectors as a generic tool for this purpose. The class of three-point techniques introduced here includes methods for discontinuity analysis, wave identification, and spatial gradient determination. Parameter vectors can be resolved fully in the spacecraft plane but further assumptions or physical constraints have to be specified to estimate the normal components. We focus on the gradient estimation problem where we check and illustrate our approach using Cluster measurements.
\end{abstract}

Keywords. Magnetospheric physics (Current systems; Instruments and techniques) - Space plasma physics (Experimental and mathematical techniques)

\section{Introduction}

The Cluster spacecraft mission triggered numerous efforts to develop and tailor special techniques for the analysis of multi-point measurements in near-Earth space plasmas. Major analysis tasks are (a) the analysis of spatial inhomogeneities through the estimation of derivative operators such as grad, curl, and div (generally referred to also as spatial gradients), (b) the examination of waves and turbulence through wave vector identification, and (c) the analysis of plasma discontinuities and shocks through the determination of boundary parameters. Four-point analysis techniques for these three problem classes were given already by Dunlop

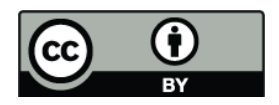

Correspondence to: J. Vogt (j.vogt@jacobs-university.de) et al. (1988). A comprehensive summary of the efforts made in the preparation phase of the Cluster mission was published by Paschmann and Daly (1998) as the first volume of the ISSI Scientific Report series. An update of this standard reference came out recently (Paschmann and Daly, 2008). More specifically, multi-spacecraft methods developed for the different analysis categories are briefly reviewed below in Sects. 3 (gradient estimation), 6.1 (wave identification), and 6.2 (boundary analysis).

Without further assumptions and constraints, four is the minimum number of measurements to resolve fully the spatio-temporal ambiguity. Due to instrument failures there are, however, several experiments (EDI, EFW, CIS) that function only on three of the four Cluster spacecraft. Suitable array techniques for the analysis of three-point measurements would allow, e.g., to estimate pressure gradients from ion measurements (CIS) or make use of electric field data (EDI, EFW) to identify wave vectors. Furthermore, threespacecraft array configurations occur regularly in the course of the THEMIS mission.

This paper offers a unifying approach to the major analysis tasks given above for the case of three-spacecraft array data. We choose a least-squares formulation to introduce and identify planar reciprocal vectors as a generic tool for three-point analyses in space plasmas (Sect. 2). The construction of a spatial gradient estimator is discussed in some detail (Sect. 3). The planar reciprocal vector approach allows to estimate the in-plane components of spatial gradients, and for the out-of-plane components we have to specify and test additional conditions, assumptions, or physical constraints. In Sect. 4, the three-spacecraft gradient estimation method is tested using Cluster magnetic field measurements where all four instruments work, and a comparison with the results of established four-spacecraft techniques is possible. The technique is also applied to data from the Cluster/CIS instruments. Accuracy, implementation, and other practical aspects of the gradient estimation scheme are addressed in

Published by Copernicus Publications on behalf of the European Geosciences Union. 
Sect. 5. Three-spacecraft analysis methods for wave vector and boundary parameter estimation are briefly discussed in Sect. 6. The wave vector estimation scheme is based on the wave surveyor technique (Vogt et al., 2008a), and the boundary analysis technique makes use of the crossing times (Harvey, 1998). We conclude in Sect. 7 with a summary of our findings and an outlook.

\section{A common approach to different analysis tasks}

The least-squares approach allows to address diverse multipoint analysis tasks such as the estimation of spatial gradients and boundary parameters (Harvey, 1998; De Keyser et al., 2005,2007 ) as well as wave vectors (Vogt et al., 2008a). In the case of four spacecraft, and if no further constraints are taken into account, the homogeneous least-squares approach to spatial gradient estimation is equivalent to the reciprocal vector method that allows to write down estimators for various analysis parameters in a very transparent way (Chanteur, 1998; Chanteur and Harvey, 1998). If the number $S$ of spacecraft is larger than four, the reciprocal vector concept can be generalized by means of a least-squares formulation (Vogt et al., 2008b). We review the basic steps of that approach first, and then extend the concept to the case of three-point measurements.

\subsection{Notation}

To ease the use of dyadic notation, vectors $\boldsymbol{a}, \boldsymbol{b}, \boldsymbol{c}, \ldots$ are always understood as column vectors. The superscript $t$ denotes the transpose which implies that, e.g., $\boldsymbol{a}^{\mathrm{t}}$ is a row vector, and the dot product of two vectors $\boldsymbol{a}$ and $\boldsymbol{b}$ can be written in the form $\boldsymbol{a} \cdot \boldsymbol{b}=\boldsymbol{a}^{\mathrm{t}} \boldsymbol{b}$. Unit vectors are indicated by $\hat{\boldsymbol{c}}$, for example, $\hat{\boldsymbol{a}}$ or $\hat{\boldsymbol{b}}$. Matrices are typeset in upright bold. The symbol I denotes the identity matrix.

The spacecraft position vectors are denoted by $\boldsymbol{r}_{\alpha}, \alpha=$ $1, \ldots, S$. Except in the general part of this Sect. 2 or when explicitly stated otherwise, the total number of spacecraft in this paper is $S=3$. Relative position vectors are written in the form $\boldsymbol{r}_{\alpha \beta}=\boldsymbol{r}_{\beta}-\boldsymbol{r}_{\alpha}$. The mean position or mesocenter of the spacecraft array is given by

$\boldsymbol{r}_{\mathrm{mc}}=\frac{1}{S} \sum_{\alpha} \boldsymbol{r}_{\alpha}$.

We call a reference frame mesocentric if the mesocenter coincides with the origin of our coordinate system. In such a frame we have $\boldsymbol{r}_{\mathrm{mc}}=\mathbf{0}$ and thus

$$
\sum_{\alpha} \boldsymbol{r}_{\alpha}=\mathbf{0}
$$

Throughout this paper, except when explicitly mentioned, coordinate systems are chosen to be mesocentric.
Of key importance for the analysis methodology introduced here is the so-called position tensor defined through

$\mathbf{R}=\sum_{\alpha} \boldsymbol{r}_{\alpha} \boldsymbol{r}_{\alpha}^{\mathrm{t}}$

\subsection{Least-squares approach to multi-point analysis}

The problems that we are addressing can all be formulated by means of cost functions of the type

$C=\sum_{\alpha}\left[\boldsymbol{m} \cdot \boldsymbol{r}_{\alpha}-d_{\alpha}\right]^{2}$

that have to be minimized with respect to a model parameter vector $\boldsymbol{m}$ for a given set of data $d_{\alpha}, \alpha=1, \ldots, S$. In the three categories of analysis tasks mentioned in the introduction, the parameter vector $\boldsymbol{m}$ and the data $d_{\alpha}$ have the following meanings.

Spatial gradient estimation. If the gradient of a scalar observable $g$ is to be estimated, the $d_{\alpha}$ are the measurements $g_{\alpha}$ at positions $\boldsymbol{r}_{\alpha}$, and the model parameter $\boldsymbol{m}=\nabla g$ is the gradient vector (Harvey, 1998; Vogt et al., 2008b).

Wave surveyor approach to wave vector identification. At a particular (angular) frequency $\omega$, the problem of identifying the wave vector $\boldsymbol{k}$ of a dominant harmonic plane wave in the observed signal can be formulated as a minimization problem where the model parameter is $\boldsymbol{m}=\boldsymbol{k}$, and the data are the phases of the (complex) eigenvector to the largest eigenvalue of the array cross spectral density matrix (Vogt et al., 2008a).

Boundary analysis using crossing times $t_{\alpha}$. A onedimensional boundary between two plasma regimes can be characterized by its normal unit vector $\hat{\boldsymbol{s}}$ and the speed $U$. The model parameter $\boldsymbol{m}=\hat{\boldsymbol{s}} / U$, and the data are the crossing times $t_{\alpha}-t_{0}$ where $t_{0}$ is the time origin (Harvey, 1998; Vogt et al., 2008b).

\subsection{The case $S \geq 4$ : Generalized reciprocal vectors}

As discussed by Harvey (1998), the minimization problem (Eq. 4) leads to the following linear equation

$\mathbf{R} \boldsymbol{m}=\sum_{\alpha} \boldsymbol{r}_{\alpha} d_{\alpha}$

for the model parameter vector $\boldsymbol{m}$, see also Vogt et al. (2008a). If the $S \geq 4$ spacecraft are not located all in one plane, the position tensor $\mathbf{R}$ can be inverted (Vogt et al., $2008 \mathrm{~b}$ ), and the solution $\boldsymbol{m}$ can be written in the form

$\boldsymbol{m}=\sum_{\alpha} \boldsymbol{q}_{\alpha} d_{\alpha}$.

where

$\boldsymbol{q}_{\alpha}=\mathbf{R}^{-1} \boldsymbol{r}_{\alpha}, \alpha=1, \ldots, S$.

E.g., the least-squares estimator for the spatial gradient $\nabla g$ of a scalar observable can be written as $\sum_{\alpha} \boldsymbol{q}_{\alpha} g_{\alpha}$. 
In the case $S=4$, the vectors $\boldsymbol{q}_{\alpha}$ coincide with the reciprocal vectors of the spacecraft tetrahedron defined through

$\boldsymbol{k}_{\alpha}=\frac{\boldsymbol{r}_{\beta \gamma} \times \boldsymbol{r}_{\beta \lambda}}{\boldsymbol{r}_{\beta \alpha} \cdot\left(\boldsymbol{r}_{\beta \gamma} \times \boldsymbol{r}_{\beta \lambda}\right)}$

(Chanteur, 1998) where $(\alpha, \beta, \gamma, \lambda)$ must be a cyclic permutation of $(1,2,3,4)$. When we wish to distinguish this case more clearly from the planar case $(S=3)$ discussed later, we call the vectors $\boldsymbol{k}_{\alpha}$ also tetrahedral reciprocal vectors. The identities $\sum_{\alpha} \boldsymbol{k}_{\alpha}=0, \sum_{\alpha} \boldsymbol{k}_{\alpha} \cdot \boldsymbol{r}_{\alpha}=3, \sum_{\alpha} \boldsymbol{k}_{\alpha} \times \boldsymbol{r}_{\alpha}=0$, and

$\sum_{\alpha} \boldsymbol{k}_{\alpha} \boldsymbol{r}_{\alpha}^{\mathrm{t}}=\mathbf{I}=\sum_{\alpha} \boldsymbol{r}_{\alpha} \boldsymbol{k}_{\alpha}^{\mathrm{t}}$

(Chanteur, 1998; Chanteur and Harvey, 1998) remain valid in the general case $S \geq 4, \boldsymbol{k}_{\alpha} \rightarrow \boldsymbol{q}_{\alpha}$, see Vogt et al. (2008b). Hence the vectors $\boldsymbol{q}_{\alpha}$ can be understood as generalized reciprocal vectors.

\subsection{The case $S=3$ : Planar reciprocal vectors}

In the case $S=3$, all spacecraft are in one plane $\mathcal{P}$. In Appendix $A$ it is shown that the position tensor $\mathbf{R}$ is singular, and its nullspace $\mathcal{N}$ is the subspace of vectors that are perpendicular to the spacecraft plane $\mathcal{P}$. For brevity, we refer to vectors in the plane $\mathcal{P}$ as planar vectors, and to those in $\mathcal{N}$ as normal vectors.

As $\mathbf{R}$ is not invertible, generalized reciprocal vectors cannot be defined on the basis of Eq. (7). Nonetheless, the solution $\boldsymbol{m}$ of Eq. (5) and hence of the minimization problem can still be written in the form

$\boldsymbol{m}=\sum_{\alpha} \boldsymbol{q}_{\alpha} d_{\alpha}$

as long as the vectors $\boldsymbol{q}_{\alpha}$ satisfy the equations

$\mathbf{R} \boldsymbol{q}_{\alpha}=\boldsymbol{r}_{\alpha}, \alpha=1,2,3$.

The solutions of the latter equations and that of Eq. (5), however, are no longer unique but determined only up to an arbitrary contribution from the nullspace $\mathcal{N}$.

To extend the reciprocal vector concept to the case $S=3$, we consider the minimum norm solution, i.e., the shortest vector $\boldsymbol{m}$ that satisfies Eq. (5). In Appendix A it is demonstrated that there is a one-to-one correspondence between minimum norm solutions and planar solutions, and that the vectors

$\boldsymbol{q}_{\alpha}=\frac{\boldsymbol{n} \times \boldsymbol{r}_{\beta \gamma}}{|\boldsymbol{n}|^{2}}, \alpha=1,2,3$,

satisfy the Eqs. (11). Here $(\alpha, \beta, \gamma)$ is the cyclic permutation of $(1,2,3)$ with $\alpha$ in the first position, and $\boldsymbol{n}$ is the normal vector defined through

$\boldsymbol{n}=\boldsymbol{r}_{12} \times \boldsymbol{r}_{13}$

Note that $\boldsymbol{n}$ is not normalized. The corresponding unit vector is denoted as $\hat{\boldsymbol{n}}=\boldsymbol{n} /|\boldsymbol{n}|$.
The vectors $\boldsymbol{q}_{\alpha}$ are obviously planar vectors, hence minimum norm solutions of Eqs. (11) and thus the generalized reciprocal vectors for the case $S=3$. For brevity, we refer to them as planar reciprocal vectors. The definition implies that their geometrical properties are completely analogous to those of the tetrahedral reciprocal vectors. E.g., $\boldsymbol{q}_{\alpha}$ is perpendicular to the side of the triangle that opposes the spacecraft at $\boldsymbol{r}_{\alpha}$, and the length $\left|\boldsymbol{q}_{\alpha}\right|$ is inversely proportional to the distance from the spacecraft to the opposing side.

The algebra of planar reciprocal vectors is presented in Appendix A. Useful identities are:

$$
\begin{aligned}
\boldsymbol{q}_{\alpha} \cdot\left(\boldsymbol{r}_{\beta}-\boldsymbol{r}_{\gamma}\right) & =\delta_{\alpha \beta}-\delta_{\alpha \gamma}, \\
\boldsymbol{q}_{\alpha} \cdot \boldsymbol{r}_{\beta} & =\delta_{\alpha \beta}-\frac{1}{3}, \\
\mathbf{I}-\hat{\boldsymbol{n}} \hat{\boldsymbol{n}}^{\mathrm{t}} & =\sum_{\alpha} \boldsymbol{q}_{\alpha} \boldsymbol{r}_{\alpha}^{\mathrm{t}}=\sum_{\alpha} \boldsymbol{r}_{\alpha} \boldsymbol{q}_{\alpha}^{\mathrm{t}}, \\
\mathbf{I}-\hat{\boldsymbol{n}} \hat{\boldsymbol{n}}^{\mathrm{t}} & =\mathbf{R Q}=\mathbf{Q R} .
\end{aligned}
$$

Here $\delta_{\alpha \beta}$ is the Kronecker symbol ( $=1$ if $\alpha=\beta$ and $=0$ otherwise), and the so-called planar reciprocal tensor is given by $\mathbf{Q}=\sum_{\alpha} \boldsymbol{q}_{\alpha} \boldsymbol{q}_{\alpha}^{\mathrm{t}}$. Finally, if the three spacecraft are part of a tetrahedral configuration, the planar reciprocal vectors $\boldsymbol{q}_{\alpha}$ are related to the tetrahedral reciprocal vectors $\boldsymbol{k}_{\alpha}$ (defined through Eq. 8) as follows:

$\boldsymbol{q}_{\alpha}=\hat{\boldsymbol{n}} \times\left(\boldsymbol{k}_{\alpha} \times \hat{\boldsymbol{n}}\right)$

for $\alpha \in\{1,2,3\}$, and $\boldsymbol{k}_{4} \| \hat{\boldsymbol{n}}$.

\section{Spatial gradient estimation}

The gradient of an observable (scalar or vector field) comprises all information about the linear part of its spatial variations. Other spatial derivatives such as the divergence or the curl of a vector field can be constructed from its gradient matrix, so it is both convenient and appropriate to refer to the problem of spatial derivative determination as gradient estimation. In the preparation phase of the Cluster mission, Dunlop et al. (1988) introduced the so-called curlometer technique to determine the curl and the divergence of a vector field from multi-spacecraft data. The least squares estimator presented by Harvey (1998) allows to take additional constraints like $\nabla \cdot \boldsymbol{B}=0$ into account. Note that without such constraints, the problem of linear gradient estimation from four-point measurements is expected to yield a unique solution (Vogt et al., 2008b). Assuming a certain degree of homogeneity in both space and time, De Keyser et al. (2007) presented a comprehensive method also based on a least squares formulation that allows to carry out detailed error analysis and an assessment of the quality of the gradient estimates, see also De Keyser (2008). A related approach was taken by Hamrin et al. (2008) to construct the so-called GALS scheme that is able to resolve convecting 
structures on spatial scales smaller than the typical spacecraft separation distance. Chanteur (1998) based his estimator on a linear interpolation scheme within the Cluster tetrahedron using barycentric coordinates, and then constructed gradient estimators on the basis of the tetrahedral reciprocal vectors defined through Eq. (8). The accuracy of linear gradient estimators was studied, e.g., by Chanteur and Harvey (1998), Robert et al. (1998a), Vogt and Paschmann (1998), and Chanteur (2000). A number of papers on Cluster data have applied the curlometer or the reciprocal vector technique to compute spatial derivatives such as grad, div, or curl. With the FGM instruments being fully operational on all four spacecraft, a number of studies to estimate the electrical current density (through the curl of the magnetic field) have been carried out in various regions of geospace such as the magnetopause (e.g., Dunlop et al., 2002), the magnetotail (Runov et al., 2005, 2006), and the ring current region (Vallat et al., 2005). See Dunlop and Eastwood (2008) and Vogt et al. (2008b) for an overview.

Without prior information or physical assumptions, only the planar component of the spatial gradient is accessible from three-point measurements in space. Using the results from the previous Sect. 2, we first formulate a planar gradient estimator before we present different options to make up for the missing information from the normal direction. For notational convenience, we make frequent use of the planar component $\nabla_{p}$ and the normal component $\nabla_{n}$ of the del (nabla) operator $\nabla$ formally defined through

$\nabla_{n}=\hat{\boldsymbol{n}}(\hat{\boldsymbol{n}} \cdot \nabla) \equiv \hat{\boldsymbol{n}} \frac{\partial}{\partial n}$

$\nabla_{p}=\nabla-\nabla_{n}$

where $\hat{\boldsymbol{n}} \cdot \nabla \equiv \partial / \partial n$ is the directional derivative along the unit vector $\hat{\boldsymbol{n}}$.

\subsection{Three-point estimation of the planar gradient}

On the basis of the concepts introduced in Sect. 2, an estimator $\tilde{\nabla}_{p} g$ for the planar component $\nabla_{p} g$ of the spatial gradient of a scalar observable $g$ is defined through

$\nabla_{p} g \simeq \tilde{\nabla}_{p} g=\sum_{\alpha} \boldsymbol{q}_{\alpha} g_{\alpha}$

where $g_{\alpha}$ are the measured values at position $\boldsymbol{r}_{\alpha}$. For a vector field $\boldsymbol{V}$, the corresponding planar gradient estimator is a matrix:

$\nabla_{p} \boldsymbol{V} \simeq \tilde{\nabla}_{p} \boldsymbol{V}=\sum_{\alpha} \boldsymbol{q}_{\alpha} \boldsymbol{V}_{\alpha}^{\mathrm{t}}$.

The divergence of a vector field is the trace of its gradient matrix. Hence the planar contribution to the divergence can be estimated through

$\nabla_{p} \cdot \boldsymbol{V} \simeq \tilde{\nabla}_{p} \cdot \boldsymbol{V}=\sum_{\alpha} \boldsymbol{q}_{\alpha} \cdot \boldsymbol{V}_{\alpha}$.
So far the analogy with the tetrahedral case is rather straightforward. In order to see which part of the curl operator can be estimated from three-point measurements, we consider the decomposition $\boldsymbol{V}=\boldsymbol{V}_{p}+\boldsymbol{V}_{n}$ of $\boldsymbol{V}$ into planar and normal components to write

$$
\begin{aligned}
\nabla \times \boldsymbol{V}= & \left(\nabla_{p}+\nabla_{n}\right) \times\left(\boldsymbol{V}_{p}+\boldsymbol{V}_{n}\right) \\
= & \nabla_{p} \times \boldsymbol{V}_{p}+\nabla_{p} \times \boldsymbol{V}_{n} \\
& +\nabla_{n} \times \boldsymbol{V}_{p}+\nabla_{n} \times \boldsymbol{V}_{n} .
\end{aligned}
$$

Here $\nabla_{p} \times \boldsymbol{V}_{p} \in \mathcal{N}, \nabla_{p} \times \boldsymbol{V}_{n} \in \mathcal{P}, \nabla_{n} \times \boldsymbol{V}_{p} \in \mathcal{P}$, and $\nabla_{n} \times$ $\boldsymbol{V}_{n}=\mathbf{0}$. The terms involving $\nabla_{p}$ can be estimated using planar reciprocal vectors. This applies to the normal component of the curl operator:

$(\nabla \times \boldsymbol{V})_{n} \simeq \tilde{\nabla}_{p} \times \boldsymbol{V}_{p}=\sum_{\alpha} \boldsymbol{q}_{\alpha} \times \boldsymbol{V}_{p, \alpha}$.

Using elementary vector calculus, the planar component can be further rearranged to yield

$$
\begin{aligned}
(\nabla \times \boldsymbol{V})_{p} & =\nabla_{p} \times \boldsymbol{V}_{n}+\nabla_{n} \times \boldsymbol{V}_{p} \\
& =\nabla_{p} V_{n} \times \hat{\boldsymbol{n}}+(\hat{\boldsymbol{n}} \cdot \nabla)\left(\hat{\boldsymbol{n}} \times \boldsymbol{V}_{p}\right)
\end{aligned}
$$

where $V_{n}=\boldsymbol{V}_{n} \cdot \hat{\boldsymbol{n}}$. The first term can be estimated through

$\nabla_{p} \times \boldsymbol{V}_{n} \simeq \tilde{\nabla}_{p} \times \boldsymbol{V}_{n}=\sum_{\alpha} \boldsymbol{q}_{\alpha} \times \boldsymbol{V}_{n, \alpha}$

or, equivalently,

$\nabla_{p} V_{n} \times \hat{\boldsymbol{n}} \simeq \tilde{\nabla}_{p} V_{n} \times \hat{\boldsymbol{n}}=\left(\sum_{\alpha} \boldsymbol{q}_{\alpha} V_{n, \alpha}\right) \times \hat{\boldsymbol{n}}$.

The second term involves the normal derivative $\hat{\boldsymbol{n}} \cdot \nabla \equiv \partial / \partial n$ that is accessible only if further information or assumptions are given.

\subsection{Different options to estimate the normal gradient}

In order to make up for the missing normal component of the gradient operator, some kind of redundancy must be present in the data. We consider three options. The gradient may be geometrically constrained to be (a) parallel or (b) perpendicular to a given vector. This approach leads to algebraic relations between the normal and planar components of the gradient. Alternatively, (c) there may be physical reasons to assume that the gradient structure is stationary in the plasma frame. In the latter case we can disregard possible temporal contributions to the variability of the signal, and attribute all non-planar variations to the changes induced by the normal component of the gradient. Of key importance in this context is the normal derivative defined through

$\frac{\partial g}{\partial n} \equiv \hat{\boldsymbol{n}} \cdot \nabla g$

where $g$ denotes a scalar observable, or one component of a vector field. Once the normal derivative is determined using 
any of the three options, the full gradient vector (or matrix) can be reconstructed:

$\nabla g=\nabla_{p} g+\frac{\partial g}{\partial n} \hat{\boldsymbol{n}}$.

Gradient parallel to a given vector. We first look at situations when the spatial gradient $\nabla g$ of an observable $g$ is parallel to a given unit vector $\hat{\boldsymbol{e}}$. E.g., in a quasimagnetohydrostatic configuration, the pressure gradient is balanced by the $\boldsymbol{J} \times \boldsymbol{B}$ force. In the case of the Cluster mission, pressure data are not available from all four spacecraft but magnetic field data are. Hence it is possible to estimate the current density, and the resulting proxy of $\boldsymbol{J} \times \boldsymbol{B}$ can be normalized to yield $\hat{\boldsymbol{e}}$. The condition $\nabla g \| \hat{\boldsymbol{e}}$ eliminates two degrees of freedom while effectively only one is left (the normal component of the gradient), so we are dealing with an overdetermined problem. The misalignment of $\nabla g$ and $\hat{\boldsymbol{e}}$ can be measured through the magnitude of the vector $\hat{\boldsymbol{e}} \times \nabla g$ that we wish to minimize. The rearrangements

$$
\begin{aligned}
|\hat{\boldsymbol{e}} \times \nabla g|^{2}= & \left|\hat{\boldsymbol{e}} \times \nabla_{p} g\right|^{2}+2\left(\hat{\boldsymbol{e}} \times \nabla_{p} g\right) \cdot\left(\hat{\boldsymbol{e}} \times \nabla_{n} g\right) \\
& +\left|\hat{\boldsymbol{e}} \times \nabla_{n} g\right|^{2} \\
= & \left|\hat{\boldsymbol{e}} \times \nabla_{p} g\right|^{2}-2\left(\hat{\boldsymbol{e}} \cdot \nabla_{p} g\right)(\hat{\boldsymbol{e}} \cdot \hat{\boldsymbol{n}}) \frac{\partial g}{\partial n} \\
& +|\hat{\boldsymbol{e}} \times \hat{\boldsymbol{n}}|^{2}\left(\frac{\partial g}{\partial n}\right)^{2}
\end{aligned}
$$

yield a quadratic equation that can be differentiated with respect to the parameter $\partial g / \partial n \equiv \hat{\boldsymbol{n}} \cdot \nabla g$. The resulting linear relation is set to zero to obtain

$$
\frac{\partial g}{\partial n}=\frac{\left(\hat{\boldsymbol{e}} \cdot \nabla_{p} g\right)(\hat{\boldsymbol{e}} \cdot \hat{\boldsymbol{n}})}{|\hat{\boldsymbol{e}} \times \hat{\boldsymbol{n}}|^{2}}
$$

The denominator $|\hat{\boldsymbol{e}} \times \hat{\boldsymbol{n}}|^{2}$ on the right-hand side of the formula should not become too small, so $\hat{\boldsymbol{e}}$ should not be too close to $\pm \hat{\boldsymbol{n}}$. We also note that the full gradient $\nabla g$ is located in the plane spanned by the vectors $\hat{\boldsymbol{n}}$ and $\nabla_{p} g$, thus $\hat{\boldsymbol{e}}$ should be close to that plane to be consistent with the constraint $\nabla g \| \hat{\boldsymbol{e}}$ imposed here.

Gradient perpendicular to a given vector. We now turn to the case $\nabla g \perp \hat{\boldsymbol{e}}$. The resulting condition

$$
0=\hat{\boldsymbol{e}} \cdot \nabla g=\hat{\boldsymbol{e}} \cdot \nabla_{p} g+(\hat{\boldsymbol{e}} \cdot \hat{\boldsymbol{n}}) \frac{\partial g}{\partial n}
$$

can be solved for the normal derivative to yield

$$
\frac{\partial g}{\partial n}=-\frac{\hat{\boldsymbol{e}} \cdot \nabla_{p} g}{\hat{\boldsymbol{e}} \cdot \hat{\boldsymbol{n}}} .
$$

Here the value of $|\hat{\boldsymbol{e}} \cdot \hat{\boldsymbol{n}}|$ can be taken as a quality indicator that should not be too small. If $|\hat{\boldsymbol{e}} \cdot \hat{\boldsymbol{n}}| \ll 1$, then the small denominator on the right-hand of the formula may introduce large errors. Geometrically, this means that the vector $\hat{\boldsymbol{e}}$ should not be too close to the spacecraft plane. Note that by construction, the geometric constraint $\nabla g \perp \hat{\boldsymbol{e}}$ can be satisfied exactly, so we cannot apply an additional consistency check as in the case $\nabla g \| \hat{\boldsymbol{e}}$.

Stationarity assumption. The third condition can be understood as a kind of dynamical rather than a geometric constraint. If the structure that carries the spatial gradient is moving with the plasma, and the velocity $\boldsymbol{U}=\boldsymbol{U}_{p}+U_{n} \hat{\boldsymbol{n}}$ of the plasma frame relative to the spacecraft array is known (e.g., using bulk velocity measurements of ions or even electrons), the temporal rate of change in the spacecraft frame is given by

$\frac{\partial g}{\partial t}=-\boldsymbol{U} \cdot \nabla g=-\left(\boldsymbol{U}_{p} \cdot \nabla_{p} g+U_{n} \frac{\partial g}{\partial n}\right)$

because the plasma frame derivative $\mathrm{d} g / \mathrm{d} t$ vanishes: $\mathrm{d} g / \mathrm{d} t=\partial g / \partial t+\boldsymbol{U} \cdot \nabla g=0$. Hence the normal derivative can be obtained from

$\frac{\partial g}{\partial n}=-\frac{1}{U_{n}}\left(\frac{\partial g}{\partial t}+\boldsymbol{U}_{p} \cdot \nabla_{p} g\right)$.

The term $\partial g / \partial t$ is the slope of the measured time series and can be estimated through a linear fit of

$g_{\mathrm{mc}}(t)=\frac{1}{3} \sum_{\alpha} g_{\alpha}(t)$

in an interval around the time of interest.

The stationarity constraint can be formulated is a slightly more general way as it builds on the condition $\mathrm{d} g / \mathrm{d} t=0$ in a reference system moving at velocity $\boldsymbol{U}$ that, however, does not have to be the plasma bulk velocity. If there is evidence that a stationary structure is moving at a constant velocity with respect to the plasma frame, the same approach would work. Note that in the four-point GALS scheme introduced by Hamrin et al. (2008), their stationarity condition uses a frame velocity $\boldsymbol{U}$ that does not need to be specified in advance but is a parameter of the optimization procedure.

Other conditions. The three constraints given above can be considered prototypes for the kind of conditions that are to be supplemented to construct the normal gradient estimates. Other types of conditions may also work. For magnetic fields, it is tempting to make use of $\nabla \cdot \boldsymbol{B}=0$ which as a single equation makes up for one degree of freedom. The problem of estimating the gradient matrix $\nabla \boldsymbol{B}$ (and a constant ambient magnetic field value, yielding 12 free parameters) from three-point measurements of magnetic field vectors $\boldsymbol{B}$ (providing 9 data points), however, is short of three degrees of freedom and would thus still be underdetermined even if the condition $\nabla \cdot \boldsymbol{B}=0$ was taken into account. Furthermore, only the diagonal of the gradient matrix $\nabla \boldsymbol{B}$ can be constrained that way but the dynamically interesting terms are the off-diagonal entries which (combined into the curl of $\boldsymbol{B})$ yield the electrical currents. Thus in this paper we have made no attempt to take advantage of the condition $\nabla \cdot \boldsymbol{B}=0$. 


\section{Cluster case studies of gradient estimation}

The three-point gradient estimation method introduced in the previous Sect. 3 is now demonstrated and validated using data from the Cluster mission. The events have been studied and published already before (Dunlop et al., 2002; Marghitu et al., 2006; Hamrin et al., 2006) and thus may serve as benchmark cases. This kind of reanalysis is meant to provide a proof of concept for and also illustrate the use of the new method.

\subsection{Comparison of three-point and four-point estimates of magnetic field gradients}

In our first case study, we use data from the Fluxgate Magnetometer (FGM) experiment that is operational on all four Cluster satellites (see, e.g., Balogh et al., 2001). Gradient estimates using any three-point subset of the spacecraft array obtained with our planar reciprocal vector approach can then be compared with the results of an established four-point method. We are using FGM measurements at spin resolution (four seconds) from the Cluster spacecraft 1, 2, and 3 (all except Cluster Tango) taken on 4 February 2001. This magnetically quiet day $\left(K_{p}=1\right)$ was studied already by Dunlop et al. (2002) using the curlometer approach. The selection of this particular subset of spacecraft was partly motivated by the resulting array geometry: the GSE position vectors of the Cluster spacecraft 1,2 , and 3 differ much less in $z$ than in their $x$ and $y$ components, hence the three-spacecraft plane is almost parallel to the GSE $(x, y)$ plane, and the three-spacecraft normal is close to the GSE $z$-axis. This kind of configuration allows to assess the analysis results more conveniently as if normal and planar contributions to the gradient enter all GSE components simultaneously. Other subsets of the Cluster array have also been tested, with minor effects on the analysis results.

The time interval considered here is 05:50-06:25 UT of 4 February 2001, when the Cluster spacecraft were located in the magnetosheath. An inbound magnetopause crossing occured later at around 07:15 UT. During this interval, the geometry of the Cluster array remained close to a regular tetrahedron. Thus gradient estimation using the four-point method of Chanteur (1998) is expected to yield small errors and can be employed as a reference for comparison with the three-spacecraft method.

As described above at the beginning of Sect. 3, we decompose the (total) gradient of the magnetic field $\nabla \boldsymbol{B}$ into a planar component $\nabla_{p} \boldsymbol{B}$ and a normal component $\nabla_{n} \boldsymbol{B}$. The planar part $\nabla_{p} \boldsymbol{B}$ can be readily obtained from the planar reciprocal vector formalism introduced in this paper, and the results are expected to be consistent with the planar projection of the four-point estimate of $\nabla \boldsymbol{B}$ because of the relationships between planar and tetrahedral reciprocal vectors given in Appendix A. The estimates of the normal derivative $\partial \boldsymbol{B} / \partial n$ and thus the normal part $\nabla_{n} \boldsymbol{B}$ of the gradient matrix depend on which of the three options is selected: the stationarity assumption, or one of the geometric conditions where the gradient (of a cartesian component of $\boldsymbol{B}$ ) is constrained to be perpendicular or parallel to a given unit vector $\hat{\boldsymbol{e}}$. Here we choose $\hat{\boldsymbol{e}}$ to be either the normalized magnetic field vector $\hat{\boldsymbol{B}}$, or the eigenvector $\hat{\boldsymbol{x}}_{\min }$ corresponding to the minimum eigenvalue of the magnetic covariance matrix. The vector $\hat{\boldsymbol{x}}_{\text {min }}$ is obtained through Minimum Variance Analysis (MVA). When a plasma boundary such as the magnetopause is crossed by spacecraft, the minimum variance direction $\hat{\boldsymbol{x}}_{\text {min }}$ can be taken as a proxy for the boundary normal. For a discussion of MVA in the context of multispacecraft missions, the reader is referred to Sonnerup and Scheible (1998). If the stationarity assumption is chosen, the plasma frame velocity $\boldsymbol{U}$ is taken to be the average ion bulk velocity measured by the Cluster Ion Spectrometry (CIS) experiment. For each of the three options, we obtained the magnetic field gradient and curl estimates for the parallel and perpendicular directions to the three-spacecraft plane using the three-spacecraft method, and compared them with the corresponding four-point estimates.

Displayed in the upper three panels of Fig. 1 are the GSE components of the $\nabla \times \boldsymbol{B}$ estimates obtained through the three-point method with the stationarity assumption, together with the results of the reference four-point method. Included are also the normal and the planar components of the threepoint curl estimates. Planar and normal flow speeds are shown in the bottom panel. The $x$ - and $y$-components of the $(\nabla \times \boldsymbol{B})_{n}$ estimate (normal component of the curl, Eq. 25) are close to zero, and the total curl estimate is given largely by the $(\nabla \times \boldsymbol{B})_{p}$ estimate (see Eq. 26). The third panel from above shows that for the $z$-component of the curl the situation is reversed, i.e., the dominant contribution to the curl estimate is $(\nabla \times \boldsymbol{B})_{n}$. The terms $\partial B_{j} / \partial t$ (see Eqs. 36 and 37) were computed using a sliding window of 20 data points corresponding to a time interval of $80 \mathrm{~s}$. We observe a good overall match of the three-point estimates and the reference four-point results except for a time interval around 06:03 UT (hatched in the figure). Here we find small values of the normal flow speed $\left|U_{n}\right|$, producing large errors in the three-point curl estimates through Eq. (36).

Figure 2 separates the planar and normal components of the gradient in the form of scatter plots where three-point estimates are drawn versus their four-point counterparts. Since most of the magnetic variation is seen in the $y$-component, estimates of $\nabla B_{y}$ are shown. Excluded from the analysis were the outliers around 06:03 UT caused by small values of the normal plasma flow speed. The $x$-component of the planar part $\left(\nabla_{p} B_{y}\right)_{x}$ and the $z$ component of the normal part $\left(\nabla_{n} B_{y}\right)_{z}$ are displayed in the upper panel and the lower panel, respectively. Linear regression analyses were performed to obtain the slopes $m$ and the Pearson correlation coefficients $R$. On the diagonal line (solid blue, slope $m=1$ ), the three-point estimates coincide with the four-point reference results. As expected, the match is perfect for the planar 


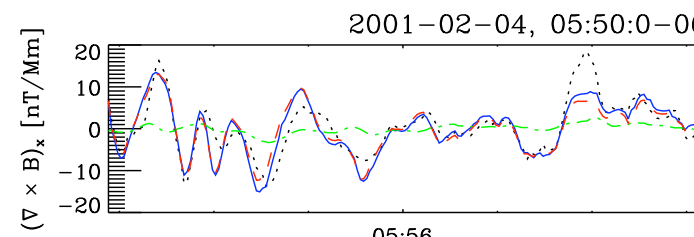

$(\nabla \times \mathrm{B})_{\mathrm{x}}$ estimates

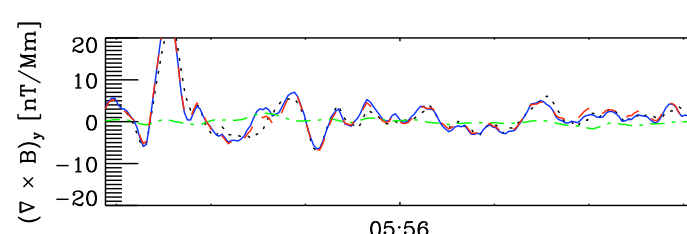

$(\nabla \times B)_{y}$ estimates

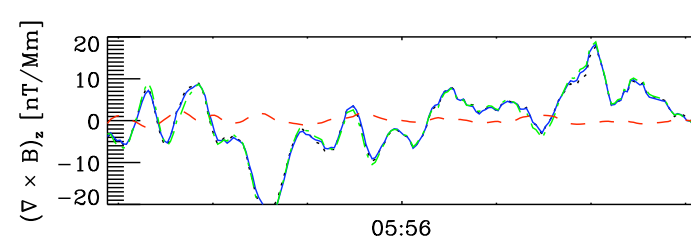

$(\nabla \times \mathrm{B})_{\mathbf{z}}$ estimates

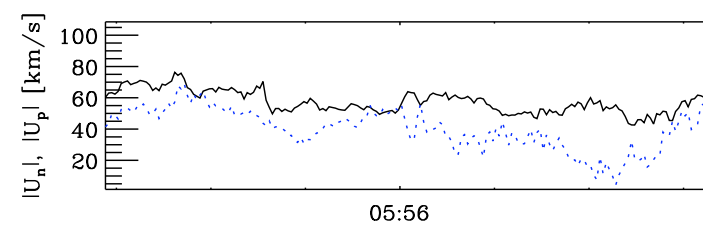

Components of the plasma flow velocity

6:25:0 UT. (Inactive Sensor: 4; Stationarity assumption)

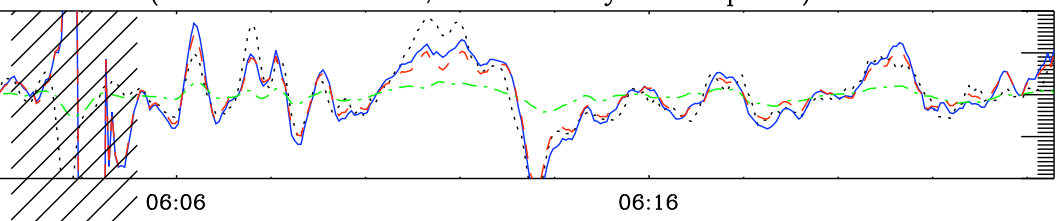

t/

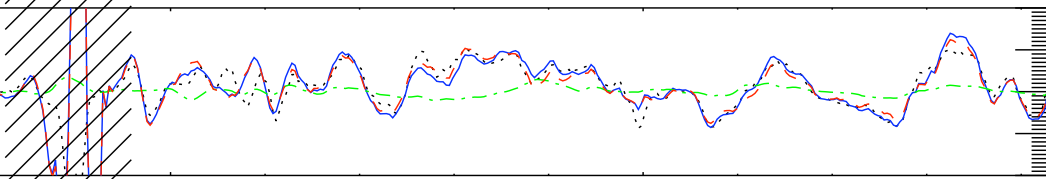

$06: 16$

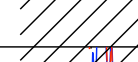

06:06

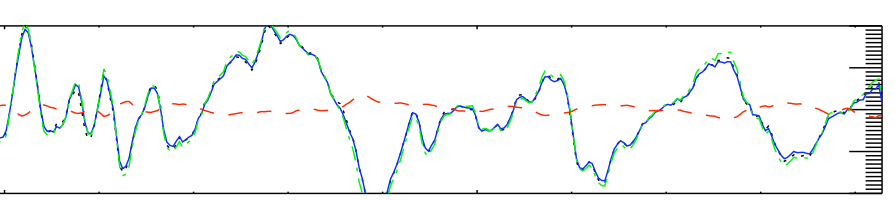

06:06

$06: 16$

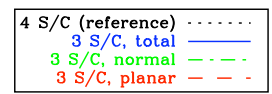

$3 \mathrm{~S} / \mathrm{C}$, total
$3 \mathrm{~S} / \mathrm{C}$, normal
$3 \mathrm{~S} / \mathrm{C}$, planar.--
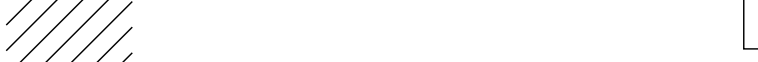

Fig. 1. 4 February 2001, 05:50-06:25 UT: GSE components of $\nabla \times \boldsymbol{B}$ (first three upper panels) as well as normal and planar components of the plasma flow velocity (lowest panel). Shown are profiles that have been averaged over $20 \mathrm{~s}$. The estimates obtained using our three-point method with the stationarity assumption (solid blue line) are compared against the four-point estimates (dotted black line). The normal component $(\nabla \times \boldsymbol{B})_{n}$ (Eq. 25) and the planar component $(\nabla \times \boldsymbol{B})_{p}$ (Eq. 26) of the curl with respect to the three-spacecraft plane are also given (dot-dashed green line and dashed red line, respectively). A significant mismatch of the three-point and four-point estimates occurs around 06:03 UT when the normal plasma flow speed takes very small values.

components of the gradient. The three-point estimates of the normal gradient component deviate from the four-point reference values typically by several $10 \%$ and tend to be smaller in magnitude.

Normal derivative estimates that result from the two geometric constraints are displayed in Figs. 3 and 4 for the same time interval as before, and also in the form of scatter plots. The panels show the z-component of the three-point estimates of $\nabla_{n} B_{y}$ versus the corresponding four-point estimates. The constraints $\nabla B_{y} \| \hat{\boldsymbol{x}}_{\text {min }}$ and $\nabla B_{y} \perp \hat{\boldsymbol{B}}$ give reasonable estimates whereas the opposite cases $\nabla B_{y} \perp \hat{\boldsymbol{x}}_{\min }$ and $\nabla B_{y} \| \hat{\boldsymbol{B}}$ yield wrong results. Since during the time interval considered here, the Cluster spacecraft are in the vicinity of the magnetopause where the minimum variance direction can be taken as a proxy for the boundary normal and thus the large-scale gradient of magnetic pressure, the assumption $\nabla B_{y} \| \hat{\boldsymbol{x}}_{\text {min }}$ makes physical sense whereas the opposite case $\nabla B_{y} \perp \hat{\boldsymbol{x}}_{\text {min }}$ does not. The condition $\nabla B_{y} \perp \hat{\boldsymbol{B}}$ means that the large-scale magnetic field gradient is perpendicular to the ambient magnetic field direction. This is consistent with the geometry of a wide range of discontinuities where the magnetic field is tangential, and it implies that the two conditions that give reasonable estimates $\left(\nabla B_{y} \| \hat{\boldsymbol{x}}_{\text {min }}\right.$ and $\left.\nabla B_{y} \perp \hat{\boldsymbol{B}}\right)$ are in fact identical. The reader is referred to studies on discontinuity analysis (e.g., Siscoe et al., 1968; Sonnerup and Scheible, 1998; Knetter et al., 2004; Bargatze et al., 2005, 2006; Haaland et al., 2006; Weimer and King, 2008) for further information.

\subsection{Estimation of pressure gradients}

In this subsection, the planar component of the gradient operator is denoted as $\nabla_{\|}$(instead of $\nabla_{p}$ as elsewhere in the paper) to avoid confusion with the (full) pressure gradient $\nabla p$, and the normal component of the gradient operator is written as $\nabla_{\perp}$. 

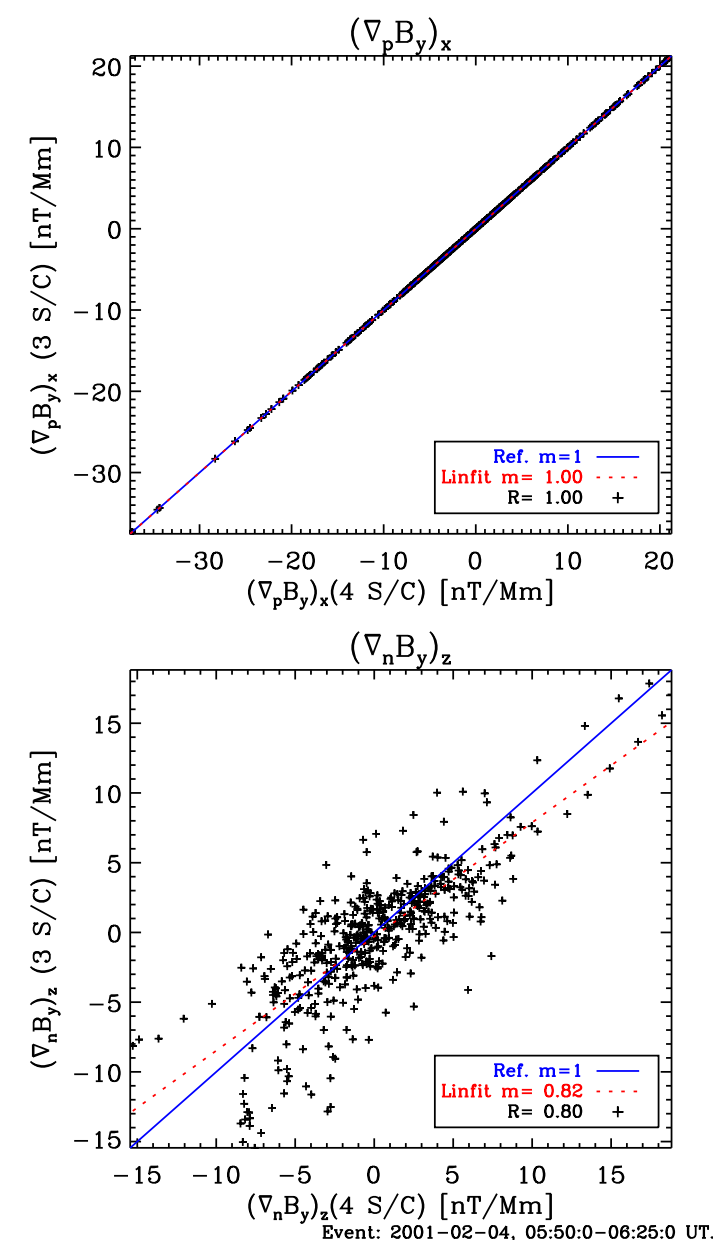

Fig. 2. 4 February 2001, 05:50-06:25 UT: Scatter plots of the $x$ component of $\nabla_{p} B_{y}$ (upper panel) and the $z$-component of $\nabla_{n} B_{y}$ (lower panel) in GSE coordinates, calculated using the stationarity assumption. Measurements during the time interval around 06:03 UT with small normal plasma flow speeds were excluded from the analysis. The horizontal axis represents the gradient component estimate using the reference four-point method. The vertical axis represents the estimate using our three-point method. The slope $m$ of the linear regression (dotted red line) and the Pearson correlation coefficient $R$ are given. The perfect match in the case of the $\nabla_{p} B_{y}$ estimate is due to the construction of the planar reciprocal vectors.

The Cluster/CIS experiment (Rème et al., 2001) provides ion data that enables the computation of macroscopic parameters, like the density, velocity, pressure, and temperature. However, because the instrument on spacecraft 2 (SC2) is not operational, only three measuring points are available, therefore the gradients cannot be estimated by four-spacecraft tools. In order to check the three-spacecraft approach, we computed the ion pressure gradient, $\nabla p$, and the work of the ion pressure forces, $W_{K}=-\boldsymbol{v} \cdot \nabla p$ (with $\boldsymbol{v}$ the ion bulk velocity), for a few concentrated generator regions (CGRs),
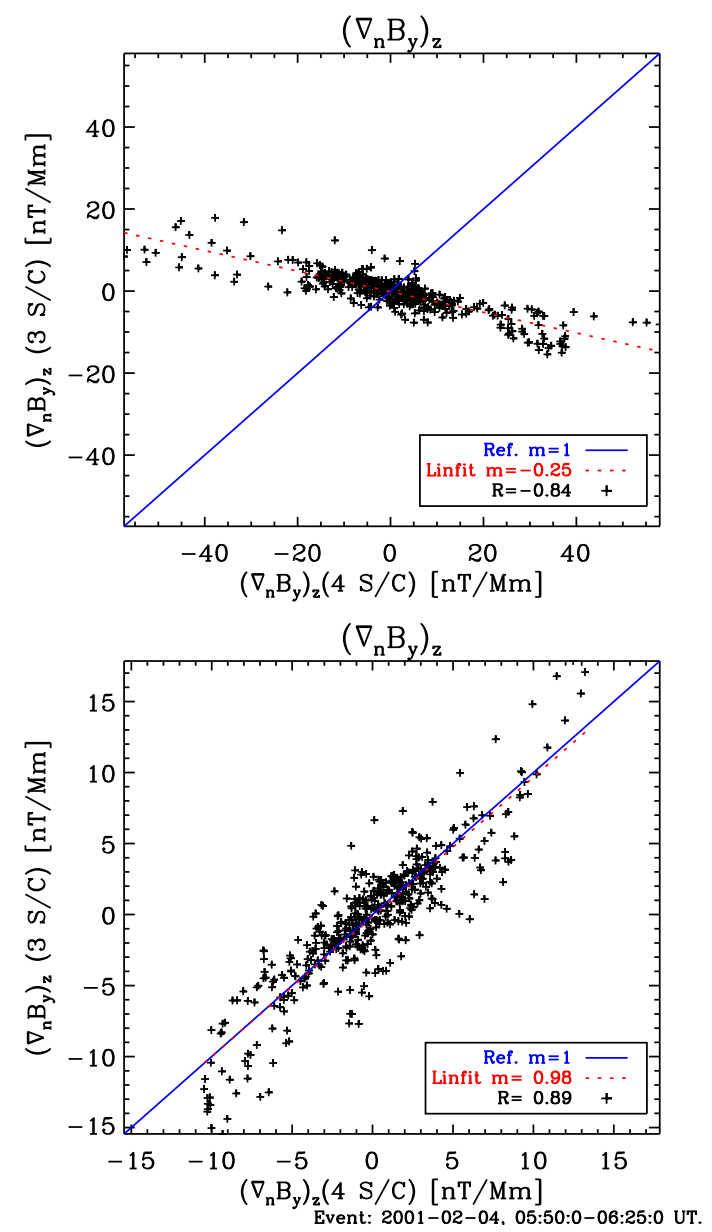

Fig. 3. 4 February 2001, 05:50-06:25 UT: Three-point normal derivative estimates $\nabla_{n} B_{y}$ using the geometric constraint $\nabla B_{y} \| \hat{\boldsymbol{e}}$ versus the corresponding four-point estimates. The unit vector $\hat{\boldsymbol{e}}$ is chosen to be the normalized ambient magnetic field direction $\hat{\boldsymbol{B}}$ (upper panel) and the minimum variance direction $\hat{\boldsymbol{x}}_{\text {min }}$ (lower panel). Notation as in Fig. 2.

formerly investigated by Marghitu et al. (2006) and Hamrin et al. (2006). In all these events, located near midnight in the plasma sheet boundary layer (PSBL), at $\sim 19 R_{E}$ geocentric distance, it was found that $\boldsymbol{E} \cdot \boldsymbol{J}<0$ (with $\boldsymbol{E}$ the electric field and $\boldsymbol{J}$ the current density). This implies that mechanical energy is locally converted into electromagnetic energy. Based on the orientation of the plasma sheet boundary, it was also possible to obtain a rough estimate of the pressure gradient and it was further inferred that $W_{K}>0$, consistent with the sense of the energy conversion.

Here we shall apply the three-spacecraft method to CGR1, the most intense CGR among those investigated in more detail. The geometry of the Cluster tetrahedron at 22:15 UT, the time of the peak $\boldsymbol{E} \cdot \boldsymbol{J}$ and $W_{K}$ (equal, respectively, to $-5 \mathrm{pW} / \mathrm{m}^{3}$ and $6 \mathrm{pW} / \mathrm{m}^{3}$ ), is presented in Fig. 5. The (SC1, $\mathrm{SC} 3, \mathrm{SC} 4)$ plane is slightly tilted with respect to the GSM 
$x$ axis $\left(\sim 15^{\circ}\right)$, and cuts the GSM $y-z$ plane along a line that makes an angle of $\sim 30^{\circ}$ with the z-axis (see also Fig. 7). In the magnetospheric tail, where the magnetic field and plasma pressure have little variation in the $x$-direction, the a priori expectation is to have the pressure gradient dominated by the component in the $y-z$-plane. In terms of the three-spacecraft approach, we expect both a planar and a normal component, whose sum is presumably normal to the plasma sheet/lobe interface.

The pressure gradients obtained by imposing the geometric constraints $\nabla p \| \boldsymbol{J} \times \boldsymbol{B}, \nabla p \perp \boldsymbol{J}$, and $\nabla p \perp \boldsymbol{B}$, as well as the respective work of the pressure forces, are shown in Fig. 6. The planar pressure gradient (c) is obtained by Eq. (21) and, as expected, its x-component is small. The three geometric constraints result in similar normal components (d, e, f), by using Eq. (32) in the first case and Eq. (34) in the other two cases. We note that the first constraint, $\nabla p \| \boldsymbol{J} \times \boldsymbol{B}$, implies that $\nabla p$ is normal to both $\boldsymbol{J}$ and $\boldsymbol{B}$, therefore the results obtained provide a consistency check for the three approaches.

In the PSBL $\boldsymbol{J}$ is often dominated by the diamagnetic current carried by ions, $\boldsymbol{J}_{d}=-\nabla p \times \boldsymbol{B} / B^{2}$, and $\nabla p, \boldsymbol{J}$, $\boldsymbol{B}$ are orthogonal to each other. A schematic configuration of the three vectors, that includes the projections of the 3spacecraft plane, and of the plasma sheet/lobe interface in the $\operatorname{GSM}(y, z)$ plane, is sketched in Fig. 7. For simplicity, $\boldsymbol{B}$ is aligned with the $\mathrm{x}$-axis, whose tilt with respect to the spacecraft plane is neglected. The pressure gradient and its components reflect the results in Fig. 6 and $\nabla p$ is assumed to be normal to the plasma sheet/lobe interface, which is close to the $\operatorname{GSM}(x, y)$-plane. The magnetic field lines thread the interface in the $\mathrm{x}$-direction, while the current is close to the $y$-direction. Note that, although the current is plotted normal to $\boldsymbol{B}$, a field-aligned component (as it is actually the case for CGR1) would not change $\nabla p$ - forced to be normal to the same $(\boldsymbol{J}, \boldsymbol{B})$ plane.

It is instructive to check in more detail the conditions under which the geometric constraint $\nabla p \| \boldsymbol{J} \times \boldsymbol{B}$ is valid. In order to have the magnetohydrostatic condition fulfilled, $\nabla p \simeq \boldsymbol{J} \times \boldsymbol{B}$, one needs to disregard the inertial term, $n m \mathrm{~d} \boldsymbol{v} / \mathrm{d} t$ (where $n$ is the particle density and $m$ the ion mass). With $m$ equal to the proton mass, $m \simeq 10^{-27} \mathrm{~kg}$, and typical values of $n \simeq 0.3 \mathrm{~cm}^{-3}, v \simeq 50 \mathrm{~km} / \mathrm{s}, j \simeq 1 \mathrm{nA} / \mathrm{m}^{2}$, $B \simeq 30 \mathrm{nT}$, one obtains that for time scales longer than $T=$ $n m v / j B \simeq 1 \mathrm{~s}$ the inertial effects are less important. Since the time resolution of the CIS measurements is, at best, $4 \mathrm{~s}$ (equal to the spin period), it appears as reasonable to assume that the magnetohydrostatic condition is fulfilled. The data used to investigate the CGRs were actually averaged over $24 \mathrm{~s}$ (roughly, the time needed by the plasma to cross the Cluster tetrahedron), therefore the magnetohydrostatic approximation is sound, and valid not only in the PSBL. The setup with $\nabla p, \boldsymbol{J}$, and $\boldsymbol{B}$ orthogonal to each other is more peculiar to the PSBL but, as discussed above, the presence of a field-aligned current is not expected to change the results.
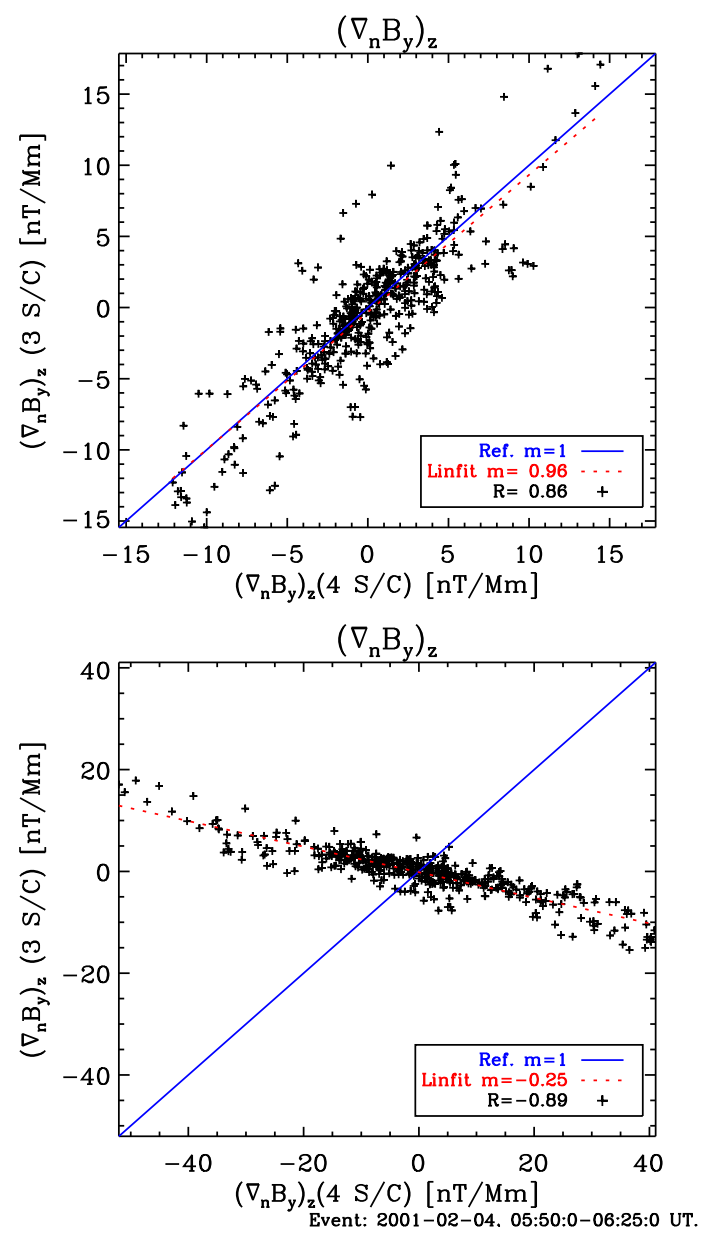

Fig. 4. Same as Fig. 3 but based on the geometric constraint $\nabla B_{y} \perp \hat{\boldsymbol{e}}\left(\right.$ instead of $\left.\nabla B_{y} \| \hat{\boldsymbol{e}}\right)$.

Consequently, the three geometric constraints should provide similar $\nabla p$ results irrespective of the Cluster location.

A different approach is based on the stationarity assumption, Eq. (36), which can be interpreted as a dynamic constraint. If the stationarity condition is indeed observed, this approach can be used also with vector operators, like div and curl, where imposing geometric constraints is less straight. On the other hand, the stationarity assumption can be difficult to fulfill on the time scales required by the experimental data. For example, with the CIS data averaged over $24 \mathrm{~s}$, if one uses a sliding window of width $w$ equal to at least 3 points in order to compute $\partial p / \partial t$ (see Eq. 37), the investigated structure should be stationary on a time scale of $72 \mathrm{~s}$ or more. In our case we checked the stationarity assumption for several values of $w \geq 3$ and the results for $w=3$ and $w=7(168 \mathrm{~s})$ are presented in Fig. 8 . The power density in the bottom panel is less negative than in Fig. 6, becoming more flat with the increase of $w$, which indicates that the stationarity assumption becomes progressively less successful 

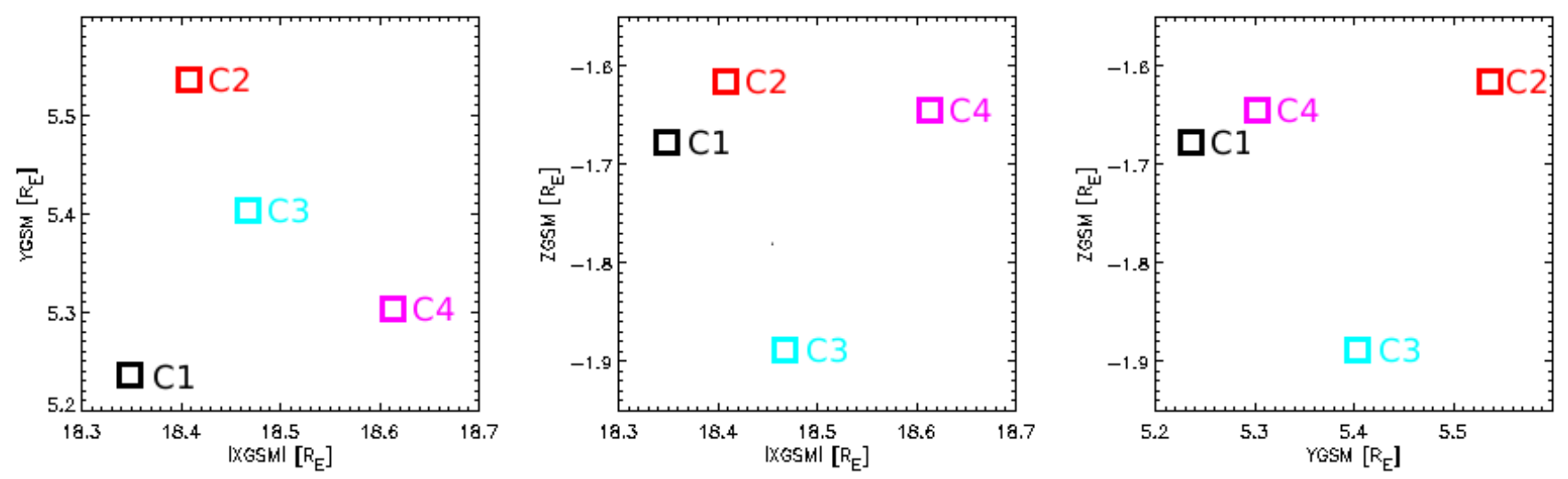

Fig. 5. Projections of the Cluster satellites in the GSM coordinate planes on 19 September 2001, at 22:15 UT.

for longer time scales. Unlike in Fig. 6, the components of $\nabla_{\perp} p$ are now negative (c, d), and $W_{K}$ has a flat maximum of $\lesssim 2 \mathrm{pW} / \mathrm{m}^{3}$ (h).

A closer examination of the stationarity assumption is provided in Fig. 9, where the various contributions to $\mathrm{d} p / \mathrm{d} t=$ $\partial p / \partial t+\boldsymbol{v} \cdot \nabla p$ are computed for two estimates of $\partial p / \partial n-$ based on the geometric constraint $\nabla p \| \boldsymbol{J} \times \boldsymbol{B}$ and on the dynamic constraint $\mathrm{d} p / \mathrm{d} t=0$. When the geometric constraint is used, $\mathrm{d} p / \mathrm{d} t$ is negative inside the CGR (e), possibly consistent with a magnetosonic wave. The $\boldsymbol{v} \cdot \nabla p$ term (d), equal to $-W_{K}$, can be compared to panel (i) in Fig. 6 (where, however, $W_{K}$ is not smoothed with $w=3$ as in Fig. 9), and to panel (h) in Fig. 8. For the geometric constraint, there is little contribution to $v \cdot \nabla p$ from the normal direction, and the positive peak of $W_{K}$ inside the CGR is related to the negative peak of $\boldsymbol{v}_{\|} \cdot \nabla_{\|} p$ (b). When the dynamic constraint is imposed, $W_{K}=-\boldsymbol{v} \cdot \nabla p=\partial p / \partial t$, which is a rather smooth function (a), slightly positive inside the CGR. In this case, the negative peak of $\boldsymbol{v}_{\|} \cdot \nabla_{\|} p$ is almost canceled by a positive peak of $\boldsymbol{v}_{\perp} \cdot \nabla_{\perp} p(\mathrm{c})$.

\section{Practical aspects of gradient estimation}

After the theoretical framework of three-point gradient estimation was formulated in Sect. 3, and analysis examples were given in Sect. 4 to demonstrate the validity of the overall concept, we now look at a few important practical issues. We start with reviewing the accuracy of four-point techniques to provide a reference for assessing the quality of the threespacecraft gradient estimation scheme.

\subsection{Error classification, accuracy of four-point estimates}

In the preparation phase of the Cluster mission, a number of studies were carried out on the accuracy of spatial gradient estimators, and to quantify the influence of geometrical factors on the analysis schemes. In the first volume of the ISSI Scientific Report series (Paschmann and Daly, 1998), chapters 12-17 deal with this subject (Harvey, 1998; Robert et al., 1998b; Chanteur, 1998; Chanteur and Harvey, 1998; Robert et al., 1998a; Vogt and Paschmann, 1998). We adopt the convention used there and classify the errors as follows.

(a) Measurement (physical) errors. These are intrinsic inaccuracies of the measurements taken by the various instruments onboard the spacecraft of the array.

(b) Positional (geometrical) errors. The positions of the spacecraft are not known precisely. These inaccuracies affect the inter-spacecraft distances and thus also the gradient estimates as they are formed by finite differences of measurements and positions. The quality of the gradient estimates is affected by the intrinsic length scale and the shape of the spacecraft configuration.

(c) Deviations from linearity. Most gradient estimation schemes implicitly assume that the observables vary linearly in space. Nonlinear variations in general, and structures with scale sizes smaller than the interspacecraft distance in particular, impose systematic errors that are difficult to assess.

In the three-spacecraft case, we are dealing with a fourth potential source or error.

(d) Uncertainties in the imposed condition. The normal component of the gradient cannot be determined directly from measurements in the three-spacecraft plane, and additional assumptions (dynamical or geometric constraints) have to be made that in general are not satisfied exactly.

Some consequences of the errors in (d) are illustrated in the next subsection 5.2, and they are more thoroughly discussed in Appendix C. We do not attempt to quantify the (negative) effects of (c) but only note that they are expected to decrease 


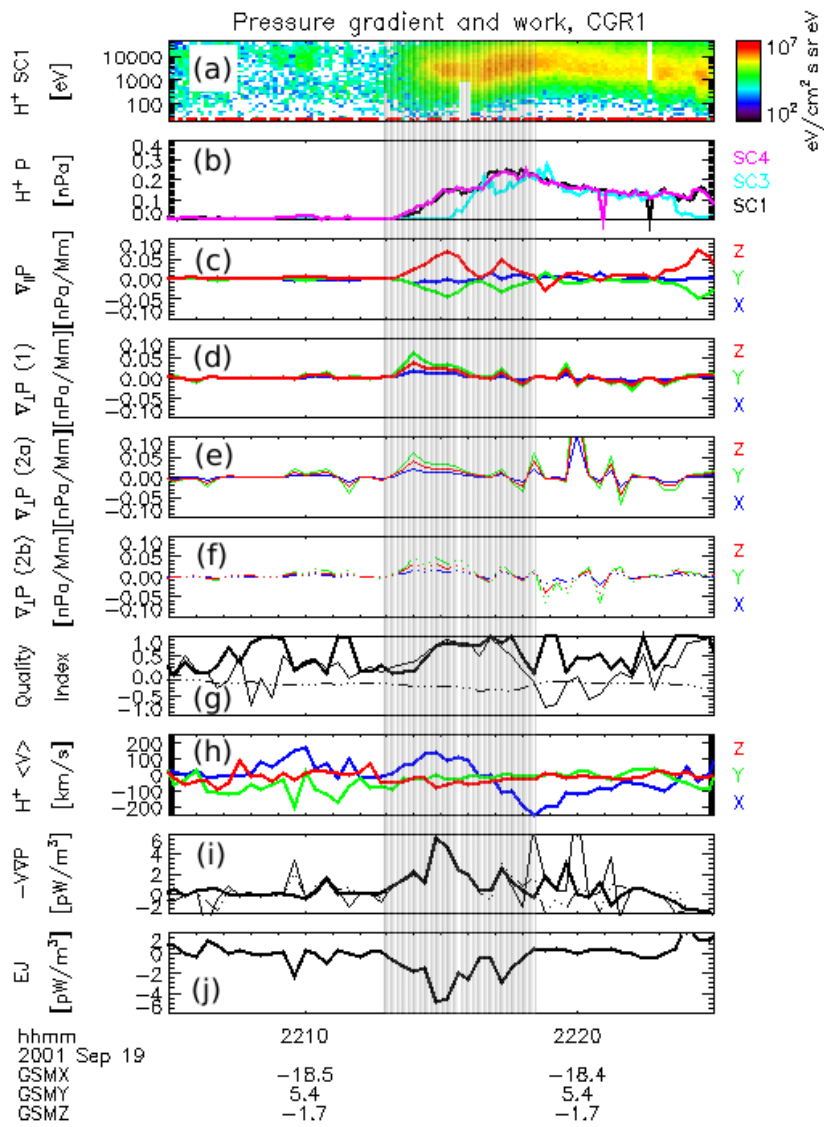

Fig. 6. Pressure gradients and the work of the pressure forces for three different geometric constraints. (a) $\mathrm{H}^{+}$energy spectrogram for SC1. (b) $\mathrm{H}^{+}$pressure for SC1 (black), SC3 (cyan), and SC4 (magenta), averaged over $24 \mathrm{~s}$. (c) Pressure gradient parallel to the 3-spacecraft plane (SC1, SC3, SC4), $\nabla_{\|} p$, computed via Eq. 21. (d, e, f) Pressure gradient normal to the 3-spacecraft plane, $\nabla_{\perp} p$, computed via Eq. (32) (d) and Eq. (34) (e, f), with the geometric constraints $\nabla p \| \boldsymbol{J} \times \boldsymbol{B}, \nabla p \perp \boldsymbol{J}$, and $\nabla p \perp \boldsymbol{B}$, respectively. (g) Quality indices $|\hat{\boldsymbol{e}} \times \hat{\boldsymbol{n}}|^{2}$ (for panel d) and $\hat{\boldsymbol{e}} \cdot \hat{\boldsymbol{n}}$ (for panels e and f), with thick, thin, and dashed-dotted line, respectively. (h) $\mathrm{H}^{+}$ velocity, averaged over spacecraft and over $24 \mathrm{~s}$. (i) The work of the pressure forces, $W_{K}=-v \cdot \nabla p$, with $\nabla p=\nabla_{\|} p+\nabla_{\perp} p$, corresponding to the three estimates of $\nabla_{\perp} p$. Same linestyles as for the quality indices. Note the peak at 22:15 and the spikes associated with low quality indices. (j) The power density, $\boldsymbol{E} \cdot \boldsymbol{J}$, indicating the energy conversion rate. The energy conversion reaches a negative peak at 22:15, simultaneous with the peak in $W_{K}$, consistent with a generator process.

with decreasing inter-spacecraft distance, and refer to the discussion in Robert et al. (1998a). We first focus on (a) and (b) that affect gradient estimation in the opposite sense: with decreasing spacecraft separation, the gradient estimates tend to get worse.

Various indicators such as the ratio of $|\nabla \cdot \boldsymbol{B}| /|\nabla \times \boldsymbol{B}|$ in magnetic field measurements, or tetrahedron geometric fac-

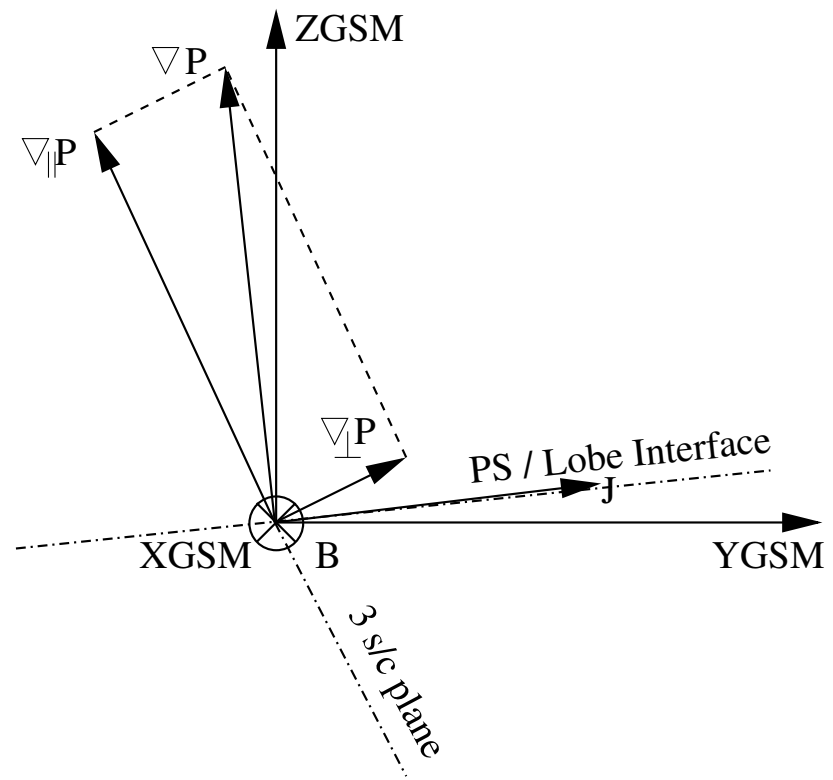

Fig. 7. Parallel, perpendicular, and total pressure gradient, together with the current density, $\boldsymbol{J}$, and the magnetic field (into the page), $\boldsymbol{B}$. The 3-spacecraft plane and the plasma sheet/lobe interface are shown as well.

tors have been suggested to assess the quality of gradient estimates but none of them has proven to work perfectly in all cases (Robert et al., 1998b,a; Chanteur and Harvey, 1998). A noteworthy approach to characterize the geometry of the Cluster tetrahedron was presented by Robert et al. (1998b). On the basis of the three eigenvalues $R_{\mathrm{vol}}^{(1)} \geq R_{\mathrm{vol}}^{(2)} \geq R_{\mathrm{vol}}^{(3)}$ of the volumetric tensor, they defined an intrinsic length scale $L_{t}$ and the two shape parameters planarity $P_{t}$ and elongation $E_{t}$ as follows:

$$
\begin{aligned}
& L_{t}=2 \sqrt{R_{\mathrm{vol}}^{(1)}}=\sqrt{R^{(1)}}, \\
& P_{t}=1-\sqrt{R_{\mathrm{vol}}^{(3)} / R_{\mathrm{vol}}^{(2)}}=1-\sqrt{R^{(3)} / R^{(2)}}, \\
& E_{t}=1-\sqrt{R_{\mathrm{vol}}^{(2)} / R_{\mathrm{vol}}^{(1)}}=1-\sqrt{R^{(2)} / R^{(1)}} .
\end{aligned}
$$

Note that the tensor $\mathbf{R}_{\mathrm{vol}}=(1 / S) \sum_{\alpha} \boldsymbol{r}_{\alpha} \boldsymbol{r}_{\alpha}^{\mathrm{t}}$ is related with the position tensor $\mathbf{R}$ through $\mathbf{R}_{\mathrm{vol}}=(1 / S) \mathbf{R}, S=4$ is the number of spacecraft in the configuration, and $L_{t}$ is a measure of the inter-spacecraft distance.

Algebraic error formulas for four-point gradient estimates are reviewed in Appendix B. To illustrate the key dependencies, we consider the gradient of a scalar variable $g$, assume the positional and measurement errors to be isotropic and mutually uncorrelated, and write the resulting covariance matrix in the following form:

$\left\langle\delta[\nabla g] \delta[\nabla g]^{\dagger}\right\rangle=\left[(\delta g)^{2}+|\nabla g|^{2}(\delta r)^{2}\right] \mathbf{K}$. 


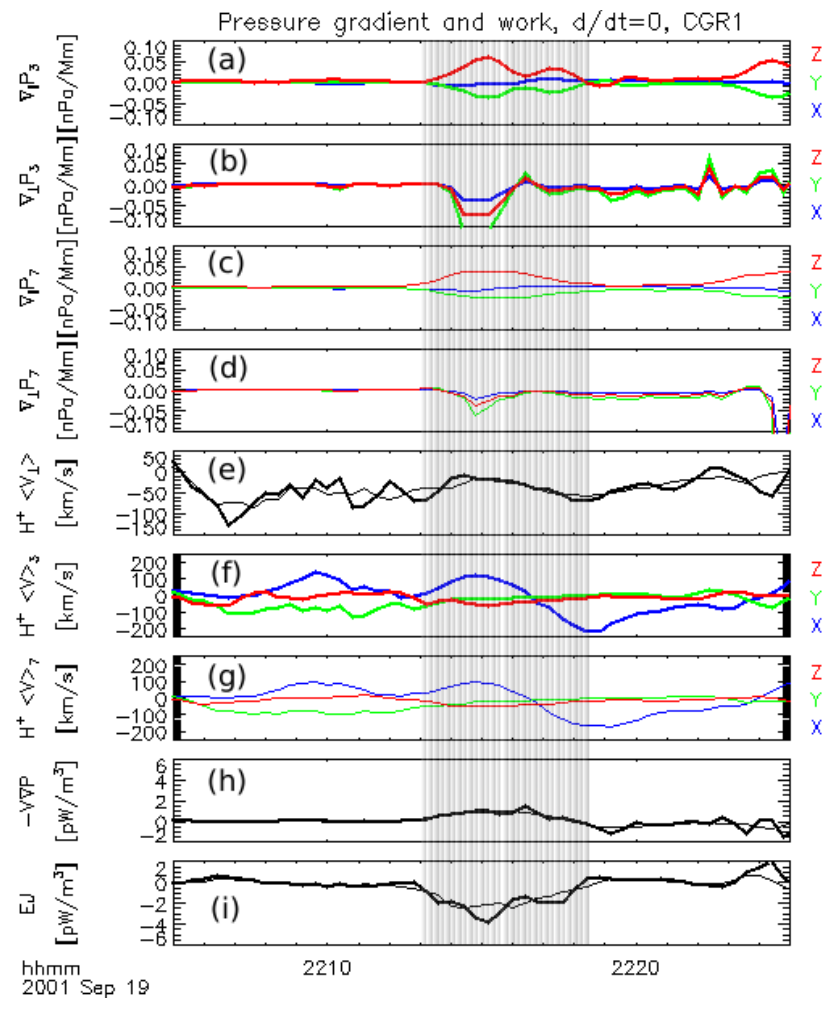

Fig. 8. Pressure gradients and the work of the pressure forces under the stationarity assumption, $\mathrm{d} p / \mathrm{d} t=0$. (a, b) Running average of the pressure gradient parallel to the 3 -spacecraft plane, $\nabla_{\|} p$, and pressure gradient normal to the 3-spacecraft plane, $\nabla_{\perp} p$, for $w=3$. (c, d) Same as (a, b), but for $w=7$. (e) The normal component of the $\mathrm{H}^{+}$velocity, proxy for the normal velocity $U_{n}$ from Eq. (36), equivalent to a quality index for the normal gradient. The thick and thin lines correspond to the estimates in the panels (b) and (d), respectively. (f, g) Running average of the $\mathrm{H}^{+}$velocity, averaged over spacecraft, for $w=3$ (f) and $w=7(\mathrm{~g})$. (h, i) $W_{K}$ and $\boldsymbol{E} \cdot \boldsymbol{J}$, with the same linestyles as in panel (e). $W_{K}$ has a flat maximum of $\lesssim 2 \mathrm{pW} / \mathrm{m}^{3}$, while $\boldsymbol{E} \cdot \boldsymbol{J}$ reaches a peak minimum of $-4 \mathrm{pW} / \mathrm{m}^{3}$ for $\tilde{w}=3$ and a flat minimum of $-2 \mathrm{pW} / \mathrm{m}^{3}$ for $w=7$. Unlike in the panels (d), (e), and (f) of Fig. 6, the components of the normal gradient are now negative $(b, d)$, reflecting the fact that the stationarity assumption is not consistent with the geometric constraints.

Here the geometry of the spacecraft tetrahedron enters only through the reciprocal tensor $\mathbf{K}$. The trace of this error formula gives the square magnitude error

$$
\left\langle|\delta \nabla g|^{2}\right\rangle=\left[(\delta g)^{2}+|\nabla g|^{2}(\delta r)^{2}\right] \operatorname{trace}(\mathbf{K})
$$

which demonstrates the meaning of the term

$\operatorname{trace}(\mathbf{K})=\sum_{\alpha=1}^{4}\left|\boldsymbol{k}_{\alpha}\right|^{2}$

as an error amplification factor due to the geometry of the spacecraft tetrahedron. The expression $\sum_{\alpha}\left|\boldsymbol{k}_{\alpha}\right|^{2}$ was identified by Vogt and Paschmann (1998) as a key factor in

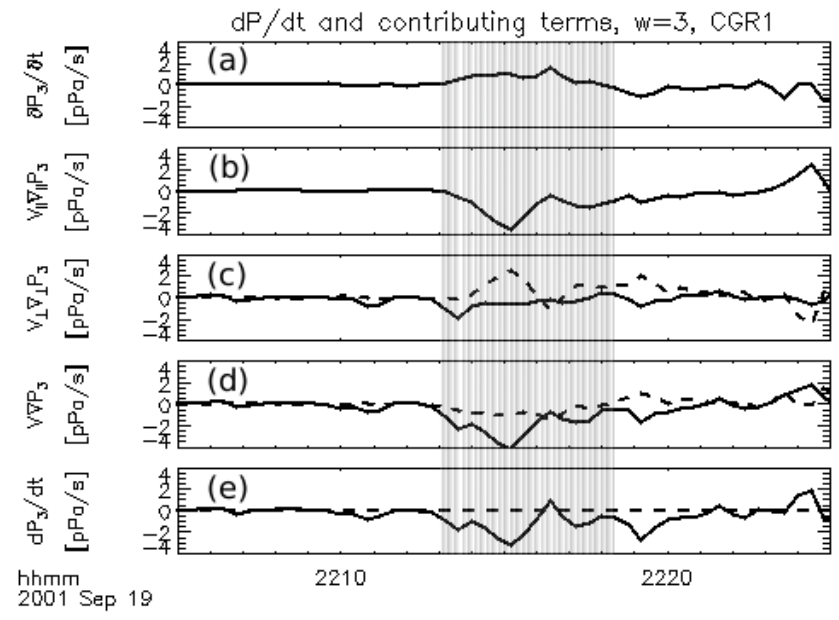

Fig. 9. Time derivative of the pressure in the plasma frame, $\mathrm{d} p / \mathrm{d} t=$ $\partial p / \partial t+\boldsymbol{v} \cdot \nabla p$, and the contributing terms, when $\partial p / \partial n$ is estimated based on $\nabla p \| \boldsymbol{J} \times \boldsymbol{B}$ (solid lines), respectively on $\mathrm{d} p / \mathrm{d} t=0$ (dashed lines). All the quantities are computed for $w=3$. (a) Time derivative in the satellite frame, $\partial p / \partial t$. (b, c) Contributions to $v \cdot \nabla p$ from the spacecraft plane, $\boldsymbol{v}_{\|} \cdot \nabla_{\|} p$, and from the normal direction, $\boldsymbol{v} \cdot \nabla_{\perp} p=v_{n} \partial p / \partial n$. (d) Total $\boldsymbol{v} \cdot \nabla p$, the sum of (b) and (c). (e) Total $\mathrm{d} p / \mathrm{d} t$, the sum of (a) and (d). As required, when stationarity is assumed $\boldsymbol{v} \cdot \nabla p=-\partial p / \partial t$ (compare the dashed line in panel (d) with panel a).

the error formulas of various spatial derivatives. Its importance was confirmed in the thorough analysis presented by Chanteur (2000) who further studied the dependency on $L_{t}, P_{t}$, and $E_{t}$. Using $\mathbf{R}^{-1}=\mathbf{K}$, one finds that

$$
\begin{aligned}
L_{t}^{2} \operatorname{trace}(\mathbf{K}) & =1+\frac{1}{\left(1-E_{t}\right)^{2}}+\frac{1}{\left(1-E_{t}\right)^{2}\left(1-P_{t}\right)^{2}} \\
& =A_{t}^{2}\left(E_{t}, P_{t}\right) .
\end{aligned}
$$

The function $A_{t}\left(E_{t}, P_{t}\right)$ is implicitly defined through the latter equation. Chanteur (2000) explained that the algebraic error formulas and the numerical approach of Robert et al. (1998a) yield consistent results. For further details the reader is referred to the original publications and to Vogt et al. (2008b).

If we combine the (isotropic) inaccuracies in position $(\delta r)$ and observable $(\delta g)$ into a single effective primary error variable defined through

$\delta_{t} g=\sqrt{(\delta g)^{2}+|\nabla g|^{2}(\delta r)^{2}}$,

then the root mean square error of the gradient magnitude can be expressed in the following convenient form

$\sqrt{\left\langle|\delta \nabla g|^{2}\right\rangle}=\frac{\delta_{t} g}{L_{K}}=\left(\frac{\delta_{t} g}{L_{t}}\right) A_{t}\left(E_{t}, P_{t}\right)$

where $L_{K}=\left(\sum_{\alpha=1}^{4}\left|\boldsymbol{k}_{\alpha}\right|^{2}\right)^{-1 / 2}$ denotes a characteristic length scale imposed by the set of tetrahedral reciprocal vectors. Since $L_{t}$ is a measure of spacecraft separation, the ratio 
$\delta_{t} g / L_{t}$ can be understood as a reference value for the inaccuracy in gradient estimation which is further amplified by the shape function $A_{t}\left(E_{t}, P_{t}\right)$. Note that for planarity values close to one, the function $A_{t}$ is well approximated as $A_{t} \simeq\left(1-E_{t}\right)^{-1}\left(1-P_{t}\right)^{-1}$.

\subsection{Accuracy of three-point gradient estimation}

To assess the quality of three-point gradient estimates, we have to consider both the planar component and the normal component. Due to the formal similarity of the gradient estimator based on tetrahedral reciprocal vectors with the estimator for the planar component in the three-spacecraft case, the quality of the latter is assessed using the same approach as for the four-point estimation scheme. Details are explained in Appendix B. The quality of the normal gradient estimate is mainly affected by the geometric or dynamical constraint of interest, see Appendix C. In this subsection we summarize the most important findings.

Accuracy of the planar component. If the measurement errors and the positional inaccuracies are mutually uncorrelated and isotropic, the covariance of the planar gradient vector of a scalar variable $g$ is given by

$\left\langle\delta\left[\nabla_{p} g\right] \delta\left[\nabla_{p} g\right]^{\mathrm{t}}\right\rangle=\left[(\delta g)^{2}+\left|\nabla_{p} g\right|^{2}(\delta r)^{2}\right] \mathbf{Q}$,

and for the square magnitude error we obtain

$\left\langle\left|\delta \nabla_{p} g\right|^{2}\right\rangle=\left[(\delta g)^{2}+\left|\nabla_{p} g\right|^{2}(\delta r)^{2}\right] \operatorname{trace}(\mathbf{Q})$.

Primary inaccuracies in spacecraft positions and measurements are thus amplified by the factor

$\operatorname{trace}(\mathbf{Q})=\sum_{\alpha=1}^{3}\left|\boldsymbol{q}_{\alpha}\right|^{2}=\frac{A_{p}^{2}\left(E_{p}\right)}{L_{p}^{2}}$

where in analogy to the four-spacecraft case an intrinsic scale $L_{p}$, the (planar) elongation $E_{p}$, and the shape function $A_{p}\left(E_{p}\right)$ of the three-spacecraft configuration are defined through

$L_{p}=\sqrt{R^{(1)}}$,

$E_{p}=1-\sqrt{R^{(2)} / R^{(1)}}$,

$A_{p}^{2}=L_{p}^{2} \operatorname{trace}(\mathbf{Q})=1+\frac{1}{\left(1-E_{p}\right)^{2}}$.

Here $R^{(1)} \geq R^{(2)} \geq R^{(3)}=0$ are the eigenvalues of the (singular) position tensor $\mathbf{R}=\sum_{\alpha=1}^{3} \boldsymbol{r}_{\alpha} \boldsymbol{r}_{\alpha}^{\mathrm{t}}$. For the root mean square error of the planar gradient magnitude we obtain

$\sqrt{\left\langle\left|\delta \nabla_{p} g\right|^{2}\right\rangle}=\frac{\delta_{p} g}{L_{Q}}=\left(\frac{\delta_{p} g}{L_{p}}\right) A_{p}\left(E_{p}\right)$

with the combined measurement/positional inaccuracy

$\delta_{p} g=\sqrt{(\delta g)^{2}+\left|\nabla_{p} g\right|^{2}(\delta r)^{2}}$, and the length scale $L_{Q}=\left(\sum_{\alpha=1}^{3}\left|\boldsymbol{q}_{\alpha}\right|^{2}\right)^{-1 / 2}$.

Accuracy of the normal component. The gradient estimate in the direction normal to the spacecraft plane is constructed not only from measurements but also from additional dynamical or geometric constraints. If these conditions are not satisfied, the quality of the normal gradient estimate will be affected. Appendix $\mathrm{C}$ makes this statement quantitative in the following sense. The unit vector $\hat{\boldsymbol{e}}$ used in the geometric constraints is assumed to deviate from the ideal choice $\hat{\boldsymbol{e}}_{\circ}$ (that fulfills the constraint exactly) by an angle $\varepsilon$. The resulting inaccuracy in the gradient estimate $\tilde{\boldsymbol{G}}=\tilde{\nabla} g$ is quantified using two error measures, namely, the relative error in magnitude

$\frac{\delta G}{G}=\frac{\tilde{G}-G}{G}$

and the directional mismatch

$\sin \Delta=\left|\frac{\tilde{\boldsymbol{G}} \times \boldsymbol{G}}{\tilde{G} G}\right|$

where $\Delta \geq 0$ is the angle between the true gradient and the estimator, and $\boldsymbol{G}=\nabla g$ denotes the true gradient. The error analysis aims at the susceptibility of the gradient estimate to small errors $(\varepsilon \ll 1)$, so the formulas are expanded, and only the leading order in $\varepsilon$ is kept. The angle $\gamma$ between the normal direction $\hat{\boldsymbol{n}}$ and the true gradient $\boldsymbol{G}=\nabla g$ turns out to be of key importance in the discussion.

If the constraint $\hat{\boldsymbol{e}} \| \boldsymbol{G}$ is applied, and $\hat{\boldsymbol{e}}$ is varied only in the plane spanned by $\boldsymbol{G}$ and $\hat{\boldsymbol{n}}$, then the magnitude estimate is most susceptible to errors in $\hat{\boldsymbol{e}}$ if $\gamma$ is small. Hence $|\hat{\boldsymbol{e}} \times \hat{\boldsymbol{n}}|$ may serve as an error indicator, see also the analysis examples in Sect. 4. The directional estimate turns out to be more robust. In fact, to lowest order in $\varepsilon$, the directional mismatch does not depend on the value of $\gamma$ at all. For the same constraint but variations of $\hat{\boldsymbol{e}}$ in the direction perpendicular to the plane spanned by $\boldsymbol{G}$ and $\hat{\boldsymbol{n}}$, the error formulas are quadratic in $\varepsilon$ which means that the gradient estimate is less affected by this class of uncertainties in $\hat{\boldsymbol{e}}$.

For the second condition $\hat{\boldsymbol{e}} \perp \boldsymbol{G}$, the unit vector $\hat{\boldsymbol{e}}$ may be freely varied in the plane perpendicular to $\boldsymbol{G}$ without affecting the geometric constraint at all, and the gradient estimate is perfectly robust. With respect to uncertainties of $\hat{\boldsymbol{e}}$ in the direction parallel to $\boldsymbol{G}$, the gradient magnitude estimate turns out to be very susceptible if $\gamma$ is small but the gradient direction may still be determined rather reliably. For this kind of geometric constraint also the orientation of $\hat{\boldsymbol{e}}$ with respect to the normal direction $\hat{\boldsymbol{n}}$ comes into play: uncertainties in $\hat{\boldsymbol{e}}$ are amplified strongly if $\hat{\boldsymbol{e}} \perp \hat{\boldsymbol{n}}$. This motivates the use of the term $|\hat{\boldsymbol{e}} \cdot \hat{\boldsymbol{n}}|$ as an error indicator in this case.

If the stationarity assumption is used as a constraint, the uncertainty in the proper choice of the velocity $\boldsymbol{U}$ is associated with a residual rate of change in the co-moving reference system which in turn can be understood as an effective error of the time derivative in the spacecraft frame. This error is amplified through the term $1 / U_{n}$ to yield the uncertainty in 
the normal derivative. Hence the gradient estimate should be interpreted with care if the plasma frame velocity vector $\boldsymbol{U}$ is close to the three-spacecraft plane. This behavior was illustrated in the analysis of the Cluster magnetic field data in Sect. 4.1.

\subsection{Implementation of the three-point gradient estimator}

To implement and use the three-point gradient estimation scheme, we recommend to proceed as follows. The procedure is given for the gradient vector $\nabla g$ of a scalar observable but applies also to the gradient matrix $\nabla \boldsymbol{V}$ of a vector field $\boldsymbol{V}$.

\section{(a) Compute the planar gradient estimate.}

From the spacecraft position vectors $\boldsymbol{r}_{\alpha}$, compute the normal vector $\hat{\boldsymbol{n}}$ (Eq. 13), the three planar reciprocal vectors $\boldsymbol{q}_{\alpha}$ (Eq. 12), and then, using the measurements $d_{\alpha}$, the planar gradient estimate $\tilde{\nabla}_{p} g$ (Eq. 21).

(b) Check the quality of the planar gradient estimate.

Combine the uncertainties in measurement $(\delta g)$ and spacecraft position $(\delta r)$ into a single effective error variable $\delta_{p} g$ (Eq. 54), and multiply with the inverse length scale $L_{O}^{-1}=\sqrt{\operatorname{trace}(\mathbf{Q})}$ to yield a proxy for the error in the planar gradient estimate. Of course, the error should only be a fraction of the actual estimate $\tilde{\nabla}_{p} g$. Alternatively, one may take the eigenvalues of the position tensor to compute the planar elongation $E_{p}$ and the intrinsic scale $L_{p}$ of the three-spacecraft configuration, and assess the quality of the planar gradient estimate through Eq. (53). Note that this kind of quality check is valid for the idealized case of isotropic and mutually uncorrelated errors in measurement and position. If more information is available (full covariance matrices), a more detailed error analysis may be carried out following the approach discussed in Appendix B.

(c) Select the constraint to be used in normal gradient estimation. This step requires careful consideration of the specific physical situation of interest. General recipes are difficult to formulate. The geometric and dynamical constraints given in Sect. 3.2 are prototypes for algebraic or differential equations reflecting some kind of redundancy in the data that can be exploited to construct a normal gradient estimate. If possible, the result should be checked for internal consistency, see below.

Examples: In the vicinity of quasi-planar discontinuities such as the magnetopause, the boundary normal is expected to be aligned with large-scale gradients. Proxies for the boundary normal vector can be the minimum variance direction of the magnetic field, or the maximum variance direction of the electric field, see Sonnerup and Scheible (1998). In the vicinity of tangential discontinuities (seemingly the dominant type in the solar wind, see Knetter et al., 2004), one may also impose that the magnetic field gradient is perpendicular to the ambient magnetic field. In the Earth's magnetotail under slow-flow conditions, the inertial terms in the magnetohydrodynamic equation of motion are relatively small, and pressure gradient estimates can be obtained from the magnetohydrostatic conditions as described in Sect. 4.2. To test the validity of the stationarity assumption, one may transform the data into the plasma frame of reference (e.g., Hamrin et al., 2008) and check if the temporal rate of change in that frame is small.

(d) Compute the normal gradient estimate. The selected constraint is numerically evaluated, and the estimate for the normal derivative $\partial g / \partial n$ is multiplied with the normal unit vector $\hat{\boldsymbol{n}}$ to yield the normal gradient estimate $\tilde{\nabla}_{n} g$.

(e) Check the quality of the normal gradient estimate. The accuracy and the significance of the normal gradient estimate depends on the quality of the planar gradient estimate, and on how exactly the imposed constraints are fulfilled. The normal gradient estimate should be taken with care if the denominators in the respective condition for the estimate of the normal derivative $\partial g / \partial n$ is small, i.e., the normal speed $\left|U_{n}\right|$ in the case of the stationarity assumption, the term $|\hat{\boldsymbol{e}} \times \hat{\boldsymbol{n}}|$ in the case of the geometric condition $\hat{\boldsymbol{e}} \| \nabla g$, or the term $|\hat{\boldsymbol{e}} \cdot \hat{\boldsymbol{n}}|$ if the condition $\hat{\boldsymbol{e}} \perp \nabla g$ is imposed.

(f) Combine normal and planar components to obtain the full gradient. Here we simply add $\tilde{\nabla}_{p} g$ and $\tilde{\nabla}_{n} g$ to determine the full gradient estimate $\tilde{\nabla} g$.

(g) Perform consistency checks. Like the four-point equivalent, the planar gradient estimation scheme exploits only the linear variation in the data which implies that the gradient is implicitly assumed to be homogeneous over the spatial extent of the spacecraft array. Thus for internal consistency of the method, the results should not vary too much over an equivalent temporal range, i.e., the time interval required by the spacecraft to cover the spatial extent. This consistency check is particularly useful for the geometric constraints that in principle allow for a point-wise (in time) reconstruction of the normal gradient component. Furthermore, the condition $\hat{\boldsymbol{e}} \| \nabla g$ may be checked a posteriori as the estimation scheme minimizes the $|\hat{\boldsymbol{e}} \times \nabla g|^{2}$ but it does not enforce $\nabla g$ to be parallel to $\hat{\boldsymbol{e}}$. This is different from the condition $\hat{\boldsymbol{e}} \perp \nabla g$ that is satisfied by construction. In the case of the stationarity assumption, the time derivative is computed from an averaged value $g_{\mathrm{mc}}(t)$. With the reconstructed gradient, the model allows to predict also the observables at the three spacecraft which may then be compared with the actual measurements. 


\subsection{Comparison with other gradient estimation schemes}

Unconstrained linear gradient estimation schemes using simultaneous measurements from four spacecraft are expected to yield the same results because the number of observations matches exactly the number of unknowns (in the scalar case, three components of the gradient vector and a constant), see also Vogt et al. (2008b). The curlometer approach (Dunlop et al., 1988), the unconstrained least squares estimator presented by Harvey (1998), and the reciprocal vector method (Chanteur, 1998) fall into this class of analysis techniques. A comparison of the latter with the planar part of our three-spacecraft method was implicitly given already in Sects. 5.1 and 5.2. Here we highlight only the role of the geometric error amplification factors that are related by

$\operatorname{trace}(\mathbf{Q})=\operatorname{trace}(\mathbf{K})-\sum_{\alpha=1}^{4}\left(\hat{\boldsymbol{n}} \cdot \boldsymbol{k}_{\alpha}\right)^{2}$,

see statement A10 in Appendix A. If in the four-point estimation scheme the term trace $(\mathbf{K})$ is too large due to planarity values $P_{t}$ that are close to one, and the resulting gradient estimate turns out to be useless, the value of $\operatorname{trace}(\mathbf{Q})$ may still be moderate enough to allow for a meaningful analysis. Then one may eliminate the measurement that corresponds to the longest of the reciprocal vectors $\boldsymbol{k}_{\alpha}$, and apply the three-point method to the remaining observations.

Instead of using at each time step individually a single set of four-spacecraft data, De Keyser et al. (2007) based their method on a sequence of multi-point observations that do not need to be synchronized (see also De Keyser, 2008). Their least squares estimator can be characterized as an inverse modeling effort that allows for rigorous error control. Measurement errors are specified on input, and they are usually assumed to vary isotropically with distance from a point in space-time, but can in principle be also anisotropic. The method was demonstrated for the four-point case but the approach is far more general, and can be applied also to threespacecraft observations. Constraints can be taken into account, and are their use is recommended in particular if a spatial direction turns out to be ill-resolved. In summary, the estimator of De Keyser et al. (2007) offers most of the components of our three-point method in a flexible and powerful framework. The three-spacecraft approach introduced in the present paper, however, should be easier to implement and to use in practice than the mathematically more complex inverse modeling apparatus, and thus may be better suited for routine analyses. Furthermore, the planar reciprocal vector formalism allows more explicit control of the decomposition into planar and normal components.

The GALS approach presented by Hamrin et al. (2008) also takes a sequence of measurements as input. The crucial ingredient of the method is the choice of a special frame of reference where the measurements are stationary. Unlike the stationarity assumption in our three-point method, Hamrin et al. (2008) do not prescribe the velocity $\boldsymbol{U}$ of this special coordinate system but determine it as part of an iterative optimization procedure. Another important variable optimized in the process is an intrinsic physical scale $(\Lambda)$ which enters a weight function in the least squares estimator, and effectively makes the method sensitive to structures that are smaller than the inter-spacecraft separation scale. The key control parameter of the GALS scheme is the length $T_{c}$ of the time interval (the so-called coherence time), so the method requires little user input. Since GALS is formulated so far only for the case of four spacecraft, a direct comparison with our planar reciprocal vector scheme is not yet possible.

\section{Wave vector and boundary parameter estimation}

Just like their tetrahedral counterparts, the planar reciprocal vectors presented in Sect. 2 allow to carry out not only spatial gradient estimation but they also facilitate wave and boundary analysis. Below we briefly sketch how to construct wave vector and boundary parameter estimators from three-point data. Details of the analysis schemes are beyond the scope of this first three-spacecraft data analysis paper, and will be left for future work.

\subsection{Wave surveyor technique for three-spacecraft data}

The problem of wave vector identification from multispacecraft data has been studied by several groups. The wave telescope introduced by Dunlop et al. (1988) and Neubauer and Glassmeier (1990) was based on a linear filter bank formulation. The $\boldsymbol{k}$-filtering technique constructed by Pinçon and co-workers (e.g., Pinçon and Lefeuvre, 1991, 1992) by means of a minimization principle is based on an estimator for the spatio-temporal power spectrum $P(\omega, \boldsymbol{k})$. The two approaches can be combined, and the two terms are now often used interchangeably (Pinçon and Motschmann, 1998; Motschmann et al., 1998; Pinçon and Glassmeier, 2008). On the basis of the $\boldsymbol{k}$-filtering/wave telescope technique, Constantinescu et al. (2007) constructed a wave detection scheme using spherical waves instead of plane waves to identify not only wave vectors but also the location of the wave source. A different class of multi-point wave analysis methods can be characterized as phase differencing techniques. Projections of the wave vector $\boldsymbol{k}$ onto the spacecraft separation vectors are estimated from phase differences of the signal measured between the corresponding pairs of sensors (e.g., Balikhin and Gedalin, 1993; Dudok de Wit et al., 1995; Walker et al., 2004). Still another wave vector estimation scheme, the wave surveyor technique, was suggested recently by Vogt et al. (2008a), and can be characterized as a direct method that does not require any peak search like the other categories of analysis techniques. It makes use of the eigenstructure of the cross spectral density matrix (e.g., Samson et al., 1990; 
Santolík et al., 2003), and is applicable to wave fields where at a particular frequency a single wave mode dominates. For further details on the different categories of multi-point wave analysis techniques, see Pinçon and Glassmeier (2008), Horbury and Osman (2008), and Vogt et al. (2008a).

The methodological framework introduced in Sect. 2 allows to construct a three-point variant of the wave surveyor technique because the algebra presented by Vogt et al. (2008a) translates directly to our case. Of course, only the planar component $\boldsymbol{k}_{p}$ of the full wave vector $\boldsymbol{k}$ is accessible but otherwise the estimation scheme remains the same. In particular, dot products of the wave vector $\boldsymbol{k}$ with the spacecraft position vectors $\boldsymbol{r}_{\alpha}$ are not affected because the position vectors are planar vectors, thus $\boldsymbol{k} \cdot \boldsymbol{r}_{\alpha}=\boldsymbol{k}_{p} \cdot \boldsymbol{r}_{\alpha}$. Since the spacecraft geometry enters the steering vector $\boldsymbol{h}(\boldsymbol{k})$ (for scalar data) or the steering matrix $\mathbf{H}(\boldsymbol{k})$ (for vector data) only through such dot products, all components of the estimator for the amplitude (polarization) vector $\boldsymbol{a}=\boldsymbol{a}(\omega)$ are accessible from three-point measurements, see Eqs. (34) and (35) in the paper of Vogt et al. (2008a).

To determine the normal component $k_{n}=\boldsymbol{k} \cdot \hat{\boldsymbol{n}}$ of the wave vector $\boldsymbol{k}$, however, additonal information is required, as in the case of spatial gradient estimation. If the observable is the magnetic field vector $\boldsymbol{B}$, we may take advantage of the condition $\nabla \cdot \boldsymbol{B}$ which implies that $\boldsymbol{k} \cdot \boldsymbol{a}=0$ for the pair of vectors $\boldsymbol{k}(\omega)$ and $\boldsymbol{a}(\omega)$ at the angular frequency $\omega$. The missing normal component of the wave vector can then be computed from

$k_{n}=-\frac{\boldsymbol{k}_{p} \cdot \boldsymbol{a}}{\hat{\boldsymbol{n}} \cdot \boldsymbol{a}}$

at each angular frequency $\omega$ of interest. Another option would be the curl-free constraint that applies, e.g., to electrostatic wave modes: $\nabla \times \boldsymbol{E}=0$. Here we obtain

$$
\left|k_{n}\right|=\frac{\left|\boldsymbol{k}_{p} \times \boldsymbol{a}\right|}{\left|\boldsymbol{a}_{p}\right|} .
$$

\subsection{Boundary analysis using crossing times}

Plasma boundaries such as discontinuities and shocks can be characterized by a set of parameters including orientation, velocity, curvature, and thickness. Depending on the physical question of interest, a variety of methods exist to estimate subsets of the boundary parameters. The celebrated minimum variance analysis (MVA) (Sonnerup and Cahill, 1967; Sonnerup and Scheible, 1998) is a single spacecraft technique that takes advantage of conservation laws to estimate the boundary normal. The discontinuity analyzer (DA) (Dunlop et al., 1988; Dunlop and Woodward, 1998) combines the MVA results of several spacecraft to analyze boundary motion and surface topology. A crossing time approach was presented by Harvey (1998). For a recent summary of the various techniques that have been applied in the context of the Cluster mission, see Sonnerup et al. (2008).
Following up on the least-squares approach to boundary parameter estimation explained in Sect. 2, planar reciprocal vectors allow to construct an estimator for the planar component $\boldsymbol{m}_{p}$ of the slowness vector $\boldsymbol{m}=\hat{\boldsymbol{s}} / U$ from the boundary crossing times $t_{\alpha}$ through

$\boldsymbol{m}_{p}=\sum_{\alpha} \boldsymbol{q}_{\alpha}\left(t_{\alpha}-t_{0}\right)$.

This information can be combined with other boundary analysis techniques in a variety of ways. E.g., if the boundary normal unit vector $\hat{\boldsymbol{s}}$ is constrained by means of singlespacecraft MVA results to be parallel or perpendicular to a given unit vector $\hat{\boldsymbol{e}}$, we can proceed as in Sect. 3.2 to obtain $m_{n}=\boldsymbol{m} \cdot \hat{\boldsymbol{n}}$, i.e., the slowness vector component perpendicular to the spacecraft plane. In the case $\hat{\boldsymbol{s}} \perp \hat{\boldsymbol{e}}$ when the boundary normal is known to lie in the plane perpendicular to a given vector $\hat{\boldsymbol{e}}$, we find

$m_{n}=-\frac{\hat{\boldsymbol{e}} \cdot \boldsymbol{m}_{p}}{\hat{\boldsymbol{e}} \cdot \hat{\boldsymbol{n}}}$.

When the boundary normal $\hat{s}$ is known to be parallel to the vector $\hat{\boldsymbol{e}}$, then

$m_{n}=\frac{\left(\hat{\boldsymbol{e}} \cdot \boldsymbol{m}_{p}\right)(\hat{\boldsymbol{e}} \cdot \hat{\boldsymbol{n}})}{|\hat{\boldsymbol{e}} \times \hat{\boldsymbol{n}}|^{2}}$.

The full slowness vector can then be constructed as $\boldsymbol{m}=$ $\boldsymbol{m}_{p}+m_{n} \hat{\boldsymbol{n}}$, and the boundary velocity $U=1 /|\boldsymbol{m}|$.

\section{Summary and outlook}

This paper introduced the set of planar reciprocal vectors as a generic and convenient tool to extract the information contained in three-point measurements. As the minimum norm solutions of a least-squares problem, planar reciprocal vectors yield robust and efficient estimators of model parameters in the spacecraft plane. Additional information, e.g., in the form of geometric constraints or physical assumptions, has to be provided to estimate the out-of-plane component of the model parameter vector.

Our approach to the analysis of three-spacecraft data allows to address major problem classes such as spatial gradient estimation, wave identification, and boundary analysis. The gradient estimation problem was chosen to demonstrate the practical applicability of the new method. Our threepoint technique and the reference four-point method perform equally well in the reconstruction of the in-plane component of the gradient vector. The quality of the out-of-plane component estimate depends on the type of additional information that is provided. For the events considered here, the outof-plane component of the gradient could be reasonably well reconstructed if the proper constraint was used. The accuracy of the new method and other practical aspects were discussed in some detail.

This study brought us from four-point measurements to the three-spacecraft analysis case where the missing information 
was compensated by one additional condition. If we go one step further and combine two conditions, we may be able to address the case of two-spacecraft configurations. However, the balance between the actual measurements and the imposed constraints would be moved towards the constraints, which makes the analysis more susceptible to uncertainties in the constraints. Another avenue could be more interesting, namely, taking measurements from three spacecraft and combining the planar reciprocal vector formalism with the GALS scheme (Hamrin et al., 2008): if in addition to the stationarity assumption a geometric constraint is taken into account, one should be able to exploit the resulting redundancy in the data and resolve structures on scales that are smaller than the inter-spacecraft separation. This and other possible extensions of the planar reciprocal vector approach (like the implementation of a three-spacecraft wave surveyor technique, see Sect. 6) are planned for the future.

\section{Appendix A}

\section{Algebra of planar reciprocal vectors}

We now formally justify the statements and identities given in Sect. 2, and include the algebra of planar reciprocal vectors for future reference. Although the results could be derived more directly from general principles of inverse theory, we gave preference to a basic linear algebra approach for reasons of completeness and internal consistency.

The number of spacecraft is $S=3$, we presume that they are not collinear, and we use a mesocentric coordinate frame. The planar subspace spanned by the three spacecraft position vectors is denoted by $\mathcal{P}$. In this context, vectors $\boldsymbol{w}$ are called normal if they are perpendicular to the spacecraft plane, i.e., $\boldsymbol{w} \perp \mathcal{P}$. A vector $\boldsymbol{v}$ is called planar if it is located in that plane: $v \in \mathcal{P}$. We are concerned with equations of the type

$$
\mathbf{R} u=b
$$

that are to be solved for $\boldsymbol{u}$. Here $\boldsymbol{b} \in \mathcal{P}$ is a given data vector, and $\mathbf{R}$ is the position tensor of the spacecraft array.

\section{A1. The nullspace $\mathcal{N}$ of the position tensor $\mathbf{R}$ is the subspace of normal vectors. In short, $\mathcal{P} \perp \mathcal{N}$.}

To demonstrate the statement, we note that if all spacecraft are located in one plane $\mathcal{P}$, then also the mesocenter that coincides with the origin of our coordinate system, and so are the three position vectors $\boldsymbol{r}_{1}, \boldsymbol{r}_{2}, \boldsymbol{r}_{3}$. Hence for any normal vector $\boldsymbol{w} \perp \mathcal{P}$ we obtain

$\boldsymbol{r}_{\alpha}^{\mathrm{t}} \boldsymbol{w}=\boldsymbol{r}_{\alpha} \cdot \boldsymbol{w}=0, \alpha=1,2,3$,

and this implies

$\mathbf{R} \boldsymbol{w}=\sum_{\alpha} \boldsymbol{r}_{\alpha} \boldsymbol{r}_{\alpha}^{\mathrm{t}} \boldsymbol{w}=0$

Therefore, normal vectors are in the nullspace of $\mathbf{R}$.
On the other hand, if we take a vector $\boldsymbol{w}$ that solves $\mathbf{R} \boldsymbol{w}=$ 0 , then also

$$
\begin{aligned}
0 & =\boldsymbol{w}^{\dagger} \mathbf{R} \boldsymbol{w}=\boldsymbol{w}^{\dagger}\left(\sum_{\alpha} \boldsymbol{r}_{\alpha} \boldsymbol{r}_{\alpha}^{\dagger}\right) \boldsymbol{w} \\
& =\sum_{\alpha} \boldsymbol{w}^{\dagger} \boldsymbol{r}_{\alpha} \boldsymbol{r}_{\alpha}^{\dagger} \boldsymbol{w}=\sum_{\alpha}\left|\boldsymbol{w}^{\dagger} \boldsymbol{r}_{\alpha}\right|^{2}
\end{aligned}
$$

which can be satisfied only if

$\boldsymbol{w}^{\dagger} \boldsymbol{r}_{\alpha} \equiv \boldsymbol{w} \cdot \boldsymbol{r}_{\alpha}=0$

for $\alpha=1,2,3$. This implies that all position vectors $\boldsymbol{r}_{\alpha}$ must be perpendicular to the vector $\boldsymbol{w}$. If the $S=3$ spacecraft are not collinear, the position vectors span the plane $\mathcal{P}$, thus we can also conclude that vectors in the nullspace $\mathcal{N}$ of $\mathbf{R}$ are normal vectors.

\section{A2. A planar solution $\boldsymbol{v}$ of Eq. (A1) is the minimum norm solution.}

This is evident from statement A1. In detail, if $v \in \mathcal{P}$ is a given particular solution, then any other solution $\boldsymbol{u} \neq \boldsymbol{v}$ can be expressed in the form $\boldsymbol{u}=\boldsymbol{v}+\boldsymbol{w}$ where $\boldsymbol{w} \neq \mathbf{0}$ is a normal vector. Since $\boldsymbol{v}$ is also a planar vector, the (Euclidean) norm of $\boldsymbol{u}$ satisfies

$|\boldsymbol{u}|^{2}=|\boldsymbol{v}|^{2}+|\boldsymbol{w}|^{2}>|\boldsymbol{v}|^{2}$

and hence $|\boldsymbol{v}|<|\boldsymbol{u}|$.

$$
\begin{aligned}
& \text { A3. The normal vector } \boldsymbol{n}=\boldsymbol{r}_{12} \times \boldsymbol{r}_{13} \text { can alter- } \\
& \text { natively be written in the forms } \boldsymbol{n}=\boldsymbol{r}_{23} \times \boldsymbol{r}_{21} \text {, } \\
& \boldsymbol{n}=\boldsymbol{r}_{31} \times \boldsymbol{r}_{32} \text {, and } \boldsymbol{n}=\boldsymbol{r}_{1} \times \boldsymbol{r}_{2}+\boldsymbol{r}_{2} \times \boldsymbol{r}_{3}+\boldsymbol{r}_{3} \times \boldsymbol{r}_{1} \text {. }
\end{aligned}
$$

The skew-symmetry of the cross-product implies that

$$
\begin{aligned}
\boldsymbol{n} & =\boldsymbol{r}_{12} \times \boldsymbol{r}_{13}=\left(\boldsymbol{r}_{2}-\boldsymbol{r}_{1}\right) \times\left(\boldsymbol{r}_{3}-\boldsymbol{r}_{1}\right) \\
& =\boldsymbol{r}_{2} \times \boldsymbol{r}_{3}-\boldsymbol{r}_{2} \times \boldsymbol{r}_{1}-\boldsymbol{r}_{1} \times \boldsymbol{r}_{3}-\boldsymbol{r}_{1} \times \boldsymbol{r}_{1} \\
& =\boldsymbol{r}_{2} \times \boldsymbol{r}_{3}+\boldsymbol{r}_{1} \times \boldsymbol{r}_{2}+\boldsymbol{r}_{3} \times \boldsymbol{r}_{1} .
\end{aligned}
$$

The cross-products $\boldsymbol{r}_{23} \times \boldsymbol{r}_{21}$ and $\boldsymbol{r}_{31} \times \boldsymbol{r}_{32}$ can be evaluated in the same way and also yield the latter expression.

$$
\begin{aligned}
& \text { A4. The planar reciprocal vectors } \boldsymbol{q}_{\alpha} \text { satisfy } \\
& \sum_{\alpha} \boldsymbol{q}_{\alpha}=\mathbf{0} \text { and also } \boldsymbol{q}_{\alpha}^{\mathrm{t}} \cdot \boldsymbol{r}_{\gamma \beta}=\delta_{\alpha \beta}-\delta_{\alpha \gamma} \text { for all } \\
& \alpha, \beta, \gamma \in\{1,2,3\} .
\end{aligned}
$$

Adding all three planar reciprocal vectors gives

$$
\sum_{\alpha} \boldsymbol{q}_{\alpha}=\frac{\boldsymbol{n}}{|\boldsymbol{n}|^{2}} \times\left(\boldsymbol{r}_{23}+\boldsymbol{r}_{31}+\boldsymbol{r}_{12}\right)=\mathbf{0} .
$$

The second relation can be rewritten as

$$
\boldsymbol{q}_{\alpha} \cdot\left(\boldsymbol{r}_{\beta}-\boldsymbol{r}_{\gamma}\right) \equiv \boldsymbol{q}_{\alpha}^{\mathrm{t}}\left(\boldsymbol{r}_{\beta}-\boldsymbol{r}_{\gamma}\right)=\delta_{\alpha \beta}-\delta_{\alpha \gamma} .
$$

We simply check this identity by going through the different possibilities for the triple of indices $(\alpha, \beta, \gamma)$. For any given $\alpha$, both sides of the equation are zero if $\beta=\gamma$. If $\alpha=\beta \neq \gamma$, 
then the right-hand side $\delta_{\alpha \beta}-\delta_{\alpha \gamma}=1$, and also the left-hand side

$$
\begin{aligned}
\boldsymbol{q}_{\alpha} \cdot \boldsymbol{r}_{\gamma \alpha} & =\frac{1}{n^{2}}\left(\boldsymbol{n} \times \boldsymbol{r}_{\gamma \mu}\right) \cdot \boldsymbol{r}_{\gamma \alpha}=\frac{1}{n^{2}} \boldsymbol{n} \cdot\left(\boldsymbol{r}_{\gamma \mu} \times \boldsymbol{r}_{\gamma \alpha}\right) \\
& =\frac{1}{n^{2}} \boldsymbol{n} \cdot \boldsymbol{n}=1
\end{aligned}
$$

where $\mu$ is chosen such that $(\alpha, \gamma, \mu)$ becomes a cyclic permutation of $(1,2,3)$. The case $\alpha=\gamma \neq \beta$ is demonstrated in a similar way. Finally, if all three indices are different, the right-hand side is zero because both terms vanish individually, and the left-hand side is zero because then $\boldsymbol{q}_{\alpha}$ is perpendicular to $\boldsymbol{r}_{\beta \gamma}=-\boldsymbol{r}_{\gamma \beta}$ by construction.

A5. The planar reciprocal vectors $\boldsymbol{q}_{\alpha}$ satisfy $\boldsymbol{q}_{\alpha} \cdot \boldsymbol{r}_{\beta}=\delta_{\alpha \beta}-\frac{1}{3}$ for all $\alpha, \beta \in\{1,2,3\}$.

The symmetry of the dot product implies that this relation can be rewritten as

$\boldsymbol{q}_{\alpha}^{\mathrm{t}} \boldsymbol{r}_{\beta} \equiv \boldsymbol{q}_{\alpha} \cdot \boldsymbol{r}_{\beta} \equiv \boldsymbol{r}_{\beta}^{\mathrm{t}} \boldsymbol{q}_{\alpha}=\delta_{\alpha \beta}-\frac{1}{3}$.

Since we are working in a mesocentric coordinate frame, we may subtract the mesocenter $\boldsymbol{r}_{\mathrm{mc}}=\mathbf{0}$ from $\boldsymbol{r}_{\beta}$ on the lefthand side of the equation to yield

$$
\begin{aligned}
\boldsymbol{q}_{\alpha}^{\mathrm{t}} \boldsymbol{r}_{\beta} & =\boldsymbol{q}_{\alpha}^{\mathrm{t}}\left(\boldsymbol{r}_{\beta}-\boldsymbol{r}_{\mathrm{mc}}\right) \\
& =\boldsymbol{q}_{\alpha}^{\mathrm{t}}\left(\frac{1}{3} \sum_{\gamma} \boldsymbol{r}_{\beta}-\frac{1}{3} \sum_{\gamma} \boldsymbol{r}_{\gamma}\right) \\
& =\frac{1}{3} \sum_{\gamma} \boldsymbol{q}_{\alpha}^{\mathrm{t}}\left(\boldsymbol{r}_{\beta}-\boldsymbol{r}_{\gamma}\right) \\
& =\frac{1}{3} \sum_{\gamma}\left(\delta_{\alpha \beta}-\delta_{\alpha \gamma}\right)=\delta_{\alpha \beta}-\frac{1}{3} .
\end{aligned}
$$

A6. The planar reciprocal vector $\boldsymbol{q}_{\alpha}$ is the minimum norm solution of the equation $\mathbf{R} \boldsymbol{q}_{\alpha}=\boldsymbol{r}_{\alpha}$.

This result is shown by means of statement A5:

$$
\begin{aligned}
\mathbf{R} \boldsymbol{q}_{\alpha} & =\sum_{\beta} \boldsymbol{r}_{\beta} \boldsymbol{r}_{\beta}^{\mathrm{t}} \boldsymbol{q}_{\alpha}=\sum_{\beta} \boldsymbol{r}_{\beta}\left(\delta_{\alpha \beta}-\frac{1}{3}\right) \\
& =\boldsymbol{r}_{\alpha}-\frac{1}{3} \sum_{\beta} \boldsymbol{r}_{\beta}=\boldsymbol{r}_{\alpha} .
\end{aligned}
$$

Since the solution vector $\boldsymbol{q}_{\alpha}$ is planar by construction, it is also the minimum norm solution according to statement A2.

$$
\begin{aligned}
& \text { A7. The planar reciprocal vectors } \boldsymbol{q}_{\alpha} \text { satisfy } \\
& \sum_{\alpha} \boldsymbol{q}_{\alpha} \boldsymbol{r}_{\alpha}^{\mathrm{t}}=\mathbf{I}-\hat{\boldsymbol{n}} \hat{\boldsymbol{n}}^{\mathrm{t}}=\sum_{\alpha} \boldsymbol{r}_{\alpha} \boldsymbol{q}_{\alpha}^{\mathrm{t}} .
\end{aligned}
$$

In order to demonstrate the identity involving the left-hand side and the central part of the equation, we let the tensors operate on $\hat{\boldsymbol{n}}$ and the set $\left\{\boldsymbol{q}_{\alpha}\right\}$ which taken together form a basis of the three-dimensional space. We obtain

$$
\left(\sum_{\alpha} \boldsymbol{q}_{\alpha} \boldsymbol{r}_{\alpha}^{\mathrm{t}}\right) \hat{\boldsymbol{n}}=\sum_{\alpha} \boldsymbol{q}_{\alpha} \boldsymbol{r}_{\alpha} \cdot \hat{\boldsymbol{n}}=0
$$

because $\boldsymbol{r}_{\alpha} \perp \hat{\boldsymbol{n}}$, and also

$$
\left(\mathbf{I}-\hat{\boldsymbol{n}} \hat{\boldsymbol{n}}^{\mathrm{t}}\right) \hat{\boldsymbol{n}}=\hat{\boldsymbol{n}}-\hat{\boldsymbol{n}}(\hat{\boldsymbol{n}} \cdot \hat{\boldsymbol{n}})=0 .
$$

Furthermore,

$$
\begin{aligned}
\left(\sum_{\alpha} \boldsymbol{q}_{\alpha} \boldsymbol{r}_{\alpha}^{\mathrm{t}}\right) \boldsymbol{q}_{\beta} & =\sum_{\alpha} \boldsymbol{q}_{\alpha} \boldsymbol{r}_{\alpha} \cdot \boldsymbol{q}_{\beta} \\
& =\sum_{\alpha} \boldsymbol{q}_{\alpha}\left[\delta_{\alpha \beta}-(1 / 3)\right] \\
& =\boldsymbol{q}_{\beta}-(1 / 3) \sum_{\alpha} \boldsymbol{q}_{\alpha} \\
& =\boldsymbol{q}_{\beta},
\end{aligned}
$$

and also

$$
\left(\mathbf{I}-\hat{\boldsymbol{n}} \hat{\boldsymbol{n}}^{\mathrm{t}}\right) \boldsymbol{q}_{\beta}=\boldsymbol{q}_{\beta}-\hat{\boldsymbol{n}} \hat{\boldsymbol{n}} \cdot \boldsymbol{q}_{\beta}=\boldsymbol{q}_{\beta}
$$

because $\hat{\boldsymbol{n}} \perp \boldsymbol{q}_{\beta}$. The second half of the identity (involving the central part and the right-hand side) is demonstrated in a similar manner by letting the tensors operate on $\hat{\boldsymbol{n}}$ and the set $\left\{\boldsymbol{r}_{\alpha}\right\}$.

Note that by taking the trace of the operators involved in identity A7 one further finds that $\sum_{\alpha} \boldsymbol{q}_{\alpha} \cdot \boldsymbol{r}_{\alpha}=2$. Since the skew-symmetric part of the operator $\mathbf{I}-\hat{\boldsymbol{n}} \hat{\boldsymbol{n}}^{\dagger}$ vanishes, one can also conclude that $\sum_{\alpha} \boldsymbol{q}_{\alpha} \times \boldsymbol{r}_{\alpha}=0$.

$$
\begin{aligned}
& \text { A8. The product of the position tensor } \mathbf{R} \text { and the } \\
& \text { so-called planar reciprocal tensor } \mathbf{Q}=\sum_{\beta} \boldsymbol{q}_{\beta} \boldsymbol{q}_{\beta}^{\dagger} \\
& \text { is given by } \mathbf{R} \mathbf{Q}=\mathbf{Q R}=\mathbf{I}-\hat{\boldsymbol{n}} \hat{\boldsymbol{n}}^{\dagger} \text {. }
\end{aligned}
$$

This is demonstrated by means of the statements A7 and A5 as follows:

$$
\begin{aligned}
\mathbf{R Q} & =\left(\sum_{\alpha} \boldsymbol{r}_{\alpha} \boldsymbol{r}_{\alpha}^{\dagger}\right)\left(\sum_{\beta} \boldsymbol{q}_{\beta} \boldsymbol{q}_{\beta}^{\dagger}\right) \\
& =\sum_{\alpha \beta} \boldsymbol{r}_{\alpha} \boldsymbol{r}_{\alpha}^{\dagger} \boldsymbol{q}_{\beta} \boldsymbol{q}_{\beta}^{\dagger} \\
& =\sum_{\alpha \beta} \boldsymbol{r}_{\alpha}\left[\delta_{\alpha \beta}-(1 / 3)\right] \boldsymbol{q}_{\beta}^{\dagger} \\
& =\sum_{\alpha} \boldsymbol{r}_{\alpha} \boldsymbol{q}_{\beta}^{\dagger}-(1 / 3)\left(\sum_{\alpha} \boldsymbol{r}_{\alpha}\right)\left(\sum_{\beta} \boldsymbol{q}_{\beta}\right)^{\dagger} \\
& =\sum_{\alpha} \boldsymbol{r}_{\alpha} \boldsymbol{q}_{\beta}^{\dagger}=\mathbf{I}-\hat{\boldsymbol{n}} \hat{\boldsymbol{n}}^{\dagger} .
\end{aligned}
$$

The identity $\mathbf{Q R}=\mathbf{I}-\hat{\boldsymbol{n}} \hat{\boldsymbol{n}}^{\mathrm{t}}$ is shown in the same way.

A9. If the three spacecraft are part of a tetrahedral configuration, the planar reciprocal vectors $\boldsymbol{q}_{\alpha}$ are related to the tetrahedral reciprocal vectors $\boldsymbol{k}_{\alpha}$ through $\boldsymbol{q}_{\alpha}=\hat{\boldsymbol{n}} \times\left(\boldsymbol{k}_{\alpha} \times \hat{\boldsymbol{n}}\right)$ for $\alpha \in\{1,2,3\}$, and $\boldsymbol{k}_{4} \| \hat{\boldsymbol{n}}$. 
The relation $\boldsymbol{k}_{4} \| \hat{\boldsymbol{n}}$ is evident from the definitions of $\boldsymbol{k}_{4}$ and $\hat{\boldsymbol{n}}$. The first relation is now shown for $\alpha=1$, the cases $\alpha=2$ and $\alpha=3$ are obtained by cyclic permutation of the indices. Since

$$
\begin{aligned}
\boldsymbol{k}_{\alpha} \times \boldsymbol{n} & =\left(\boldsymbol{k}_{1} \times \hat{\boldsymbol{n}}\right)(\hat{\boldsymbol{n}} \cdot \boldsymbol{n})-\hat{\boldsymbol{n}}\left[\left(\boldsymbol{k}_{1} \times \hat{\boldsymbol{n}}\right) \cdot \boldsymbol{n}\right] \\
& =\left[\hat{\boldsymbol{n}} \times\left(\boldsymbol{k}_{1} \times \hat{\boldsymbol{n}}\right)\right] \times \boldsymbol{n}
\end{aligned}
$$

(note that $\boldsymbol{a} \times(\boldsymbol{b} \times \boldsymbol{c})=\boldsymbol{b}(\boldsymbol{a} \cdot \boldsymbol{c})-\boldsymbol{c}(\boldsymbol{a} \cdot \boldsymbol{b})$ for arbitrary triples of vectors $\boldsymbol{a}, \boldsymbol{b}, \boldsymbol{c})$ and both sides of the relation to be proven are planar vectors, it is sufficient to demonstrate that

$\boldsymbol{q}_{1} \times \boldsymbol{n}=\boldsymbol{k}_{1} \times \boldsymbol{n}$.

After multiplication with

$$
\begin{aligned}
V & =\boldsymbol{r}_{21} \cdot\left(\boldsymbol{r}_{23} \times \boldsymbol{r}_{24}\right)=\left(\boldsymbol{r}_{21} \times \boldsymbol{r}_{23}\right) \cdot \boldsymbol{r}_{24} \\
& =-\left(\boldsymbol{r}_{23} \times \boldsymbol{r}_{21}\right) \cdot \boldsymbol{r}_{24}=-\boldsymbol{n} \cdot \boldsymbol{r}_{24},
\end{aligned}
$$

we are left with the relation

$\left(\boldsymbol{n} \cdot \boldsymbol{r}_{24}\right)\left(\boldsymbol{q}_{1} \times \boldsymbol{n}\right)=\left(\boldsymbol{r}_{23} \times \boldsymbol{r}_{24}\right) \times \boldsymbol{n}$

that will be shown now. The right-hand side can be rearranged to yield

$$
\begin{aligned}
-\left[\boldsymbol{n} \times\left(\boldsymbol{r}_{23} \times \boldsymbol{r}_{24}\right)\right] & =-\left[\boldsymbol{r}_{23}\left(\boldsymbol{n} \cdot \boldsymbol{r}_{24}\right)-\boldsymbol{r}_{24}\left(\boldsymbol{n} \cdot \boldsymbol{r}_{23}\right)\right] \\
& =-\boldsymbol{r}_{23}\left(\boldsymbol{n} \cdot \boldsymbol{r}_{24}\right) .
\end{aligned}
$$

The left-hand side can be reduced to the same result:

$$
\begin{aligned}
\boldsymbol{q}_{1} \times \boldsymbol{n} & =-\frac{1}{|\boldsymbol{n}|^{2}}\left[\boldsymbol{n} \times\left(\boldsymbol{n} \times \boldsymbol{r}_{23}\right)\right] \\
& =-\frac{1}{|\boldsymbol{n}|^{2}}\left[\boldsymbol{r}_{23}|\boldsymbol{n}|^{2}-\boldsymbol{n}\left(\boldsymbol{r}_{23} \cdot \boldsymbol{n}\right)\right] \\
& =-\boldsymbol{r}_{23}
\end{aligned}
$$

which completes the proof.

A10. If the three-spacecraft array with planar reciprocal vectors $\boldsymbol{q}_{\alpha}$ is part of a tetrahedral configuration with tetrahedral reciprocal vectors $\boldsymbol{k}_{\alpha}$, then

$$
\sum_{\alpha=1}^{3}\left|\boldsymbol{q}_{\alpha}\right|^{2}=\sum_{\alpha=1}^{4}\left|\boldsymbol{k}_{\alpha}\right|^{2}-\sum_{\alpha=1}^{4}\left(\hat{\boldsymbol{n}} \cdot \boldsymbol{k}_{\alpha}\right)^{2}
$$

or, equivalently,

$$
\operatorname{trace}(\mathbf{Q})=\operatorname{trace}(\mathbf{K})-\sum_{\alpha=1}^{4}\left(\hat{\boldsymbol{n}} \cdot \boldsymbol{k}_{\alpha}\right)^{2} .
$$

To prove this statement, we begin with A9:

$\boldsymbol{q}_{\alpha}=\hat{\boldsymbol{n}} \times\left(\boldsymbol{k}_{\alpha} \times \hat{\boldsymbol{n}}\right)=\boldsymbol{k}_{\alpha}-\left(\hat{\boldsymbol{n}} \cdot \boldsymbol{k}_{\alpha}\right) \hat{\boldsymbol{n}}$ for $\alpha \in\{1,2,3\}$, and thus

$$
\begin{gathered}
\mathbf{Q}=\sum_{\alpha=1}^{3} \boldsymbol{q}_{\alpha} \boldsymbol{q}_{\alpha}^{\mathrm{t}} \\
=\sum_{\alpha=1}^{3}\left[\boldsymbol{k}_{\alpha}-\left(\hat{\boldsymbol{n}} \cdot \boldsymbol{k}_{\alpha}\right) \hat{\boldsymbol{n}}\right]\left[\boldsymbol{k}_{\alpha}^{\mathrm{t}}-\left(\hat{\boldsymbol{n}} \cdot \boldsymbol{k}_{\alpha}\right) \hat{\boldsymbol{n}}^{\mathrm{t}}\right] \\
=\sum_{\alpha=1}^{3}\left\{\boldsymbol{k}_{\alpha} \boldsymbol{k}_{\alpha}^{\mathrm{t}}-\left(\hat{\boldsymbol{n}} \cdot \boldsymbol{k}_{\alpha}\right)\left[\hat{\boldsymbol{n}} \boldsymbol{k}_{\alpha}^{\mathrm{t}}+\boldsymbol{k}_{\alpha} \hat{\boldsymbol{n}}^{\mathrm{t}}\right]\right. \\
\left.\quad+\left(\hat{\boldsymbol{n}} \cdot \boldsymbol{k}_{\alpha}\right)^{2} \hat{\boldsymbol{n}} \hat{\boldsymbol{n}}^{\mathrm{t}}\right\} \\
=\mathbf{K}-\boldsymbol{k}_{4} \boldsymbol{k}_{4}^{\mathrm{t}}-\sum_{\alpha=1}^{3}\left(\hat{\boldsymbol{n}} \cdot \boldsymbol{k}_{\alpha}\right)\left[\hat{\boldsymbol{n}} \boldsymbol{k}_{\alpha}^{\mathrm{t}}+\boldsymbol{k}_{\alpha} \hat{\boldsymbol{n}}^{\mathrm{t}}\right] \\
\quad+\sum_{\alpha=1}^{3}\left(\hat{\boldsymbol{n}} \cdot \boldsymbol{k}_{\alpha}\right)^{2} \hat{\boldsymbol{n}} \hat{\boldsymbol{n}}^{\mathrm{t}} .
\end{gathered}
$$

Noting that the trace of a dyad $\boldsymbol{a} \boldsymbol{b}^{\mathrm{t}}$ is the scalar product of the two vectors, i.e., $\operatorname{trace}\left(\boldsymbol{a} \boldsymbol{b}^{\mathrm{t}}\right)=\boldsymbol{a}^{\mathrm{t}} \boldsymbol{b}=\boldsymbol{a} \cdot \boldsymbol{b}$, it is straightforward to form the traces of the matrices on both sides of the equation:

$$
\begin{aligned}
\operatorname{trace}(\mathbf{Q})= & \operatorname{trace}(\mathbf{K})-\left|\boldsymbol{k}_{4}\right|^{2}-2 \sum_{\alpha=1}^{3}\left(\hat{\boldsymbol{n}} \cdot \boldsymbol{k}_{\alpha}\right)^{2} \\
& +\sum_{\alpha=1}^{3}\left(\hat{\boldsymbol{n}} \cdot \boldsymbol{k}_{\alpha}\right)^{2} \hat{\boldsymbol{n}} \hat{\boldsymbol{n}}^{\mathrm{t}} \\
= & \operatorname{trace}(\mathbf{K})-\left|\boldsymbol{k}_{4}\right|^{2}-\sum_{\alpha=1}^{3}\left(\hat{\boldsymbol{n}} \cdot \boldsymbol{k}_{\alpha}\right)^{2} .
\end{aligned}
$$

Since $\boldsymbol{k}_{4} \| \hat{\boldsymbol{n}}$, we have $\left|\boldsymbol{k}_{4}\right|^{2}=\left(\hat{\boldsymbol{n}} \cdot \boldsymbol{k}_{4}\right)^{2}$ and thus

$$
\operatorname{trace}(\mathbf{Q})=\operatorname{trace}(\mathbf{K})-\sum_{\alpha=1}^{4}\left(\hat{\boldsymbol{n}} \cdot \boldsymbol{k}_{\alpha}\right)^{2} .
$$

\section{Appendix B}

\section{Accuracy of planar gradient estimation}

Using planar reciprocal vectors $\boldsymbol{q}_{\alpha}$, the planar components of the spatial gradients of a scalar observable $g$ and a vector field $\boldsymbol{V}$ can be estimated through

$$
\begin{gathered}
\nabla_{p} g \simeq \tilde{\nabla}_{p} g=\sum_{\alpha} \boldsymbol{q}_{\alpha} g_{\alpha}, \\
\nabla_{p} \boldsymbol{V} \simeq \tilde{\nabla}_{p} \boldsymbol{V}=\sum_{\alpha} \boldsymbol{q}_{\alpha} \boldsymbol{V}_{\alpha}^{\mathrm{t}} .
\end{gathered}
$$

The estimators are of the same functional form as in the fourpoint case studied in detail by several authors in the first volume of the ISSI Scientific Report series (Paschmann and Daly, 1998). Algebraic accuracy analyses were carried out by Chanteur $(1998,2000)$, Chanteur and Harvey (1998), and 
also by Vogt and Paschmann (1998). Many of their error estimates translate directly to the three-spacecraft case considered here as they are based on the list of algebraic relationships for reciprocal vectors that are valid in both the tetrahedral and the planar case. We expect, e.g., the inverse length scale

$L_{Q}^{-1}=\sqrt{\sum_{\alpha=1}^{3}\left|\boldsymbol{q}_{\alpha}\right|^{2}}$

to play a key role in the error analysis of three-point gradient estimates because the corresponding four-point expression

$L_{K}^{-1}=\sqrt{\sum_{\alpha=1}^{4}\left|\boldsymbol{k}_{\alpha}\right|^{2}}$

allows to write down handy formulas for directionally averaged errors of various spatial derivatives in the presence of isotropic and uncorrelated measurements inaccuracies (Vogt and Paschmann, 1998; Chanteur, 2000; Vogt et al., 2008b).

One key step in the error estimation process, however, requires special attention and careful interpretation. We adopt the approach taken by Chanteur (1998, Sects. 14.3.1 and 14.3.2) who based part of his analysis on the equation

$\sum_{\alpha}\left(\delta \boldsymbol{k}_{\alpha} \boldsymbol{r}_{\alpha}^{\mathrm{t}}+\boldsymbol{k}_{\alpha} \delta \boldsymbol{r}_{\alpha}^{\mathrm{t}}\right)=0$

which is the (linear) variation of

$\sum_{\alpha} \boldsymbol{k}_{\alpha} \boldsymbol{r}_{\alpha}^{\mathrm{t}}=\mathbf{I}=\mathrm{const}$.

The right-hand side of the corresponding expression in the planar case, namely,

$\sum_{\alpha} \boldsymbol{q}_{\alpha} \boldsymbol{r}_{\alpha}^{\mathrm{t}}=\sum_{\alpha} \boldsymbol{r}_{\alpha} \boldsymbol{q}_{\alpha}^{\mathrm{t}}=\mathbf{I}-\hat{\boldsymbol{n}} \hat{\boldsymbol{n}}^{\mathrm{t}}$

is constant only if the vector $\hat{\boldsymbol{n}}$, or, equivalently, the orientation of the three-spacecraft plane does not change in the variational process. So for the sake of simplicity, and to be able to make direct use of the results obtained for the fourpoint case, we adopt the idealized but somewhat unrealistic assumption that the three spacecraft position vectors vary only in the plane perpendicular to the vector $\hat{\boldsymbol{n}}$.

Using Eq. (B5) and the algebra of reciprocal vectors, Chanteur (1998) derived the following general expression for the covariance matrix of reciprocal vectors:

$\left\langle\delta \boldsymbol{k}_{\alpha} \delta \boldsymbol{k}_{\beta}^{\mathrm{t}}\right\rangle=\sum_{\mu=1}^{4} \sum_{\nu=1}^{4}\left(\boldsymbol{k}_{\alpha}^{\mathrm{t}}\left\langle\delta \boldsymbol{r}_{\mu} \delta \boldsymbol{r}_{\nu}^{\mathrm{t}}\right\rangle \boldsymbol{k}_{\beta}\right) \mathbf{K}$,

see Eq. (14.26) in Chanteur (1998). The covariance tensor $\left\langle\delta G_{i j} \delta G_{m n}\right\rangle$ of the linear estimator $\mathbf{G}=\sum_{\alpha} \boldsymbol{k}_{\alpha} \boldsymbol{V}_{\alpha}^{\mathrm{t}}$ for the gradient of a vector field $\boldsymbol{V}$ is given in the same publication (Eq. 14.30):

$$
\begin{aligned}
\left\langle\delta G_{i j} \delta G_{m n}\right\rangle=\sum_{\alpha=1}^{4} \sum_{\beta=1}^{4}\left(\left\langle\delta V_{\alpha, i} \delta V_{\beta, m}\right\rangle k_{\alpha, j} k_{\beta, n}\right. \\
\left.+\left\langle k_{\alpha, j} k_{\beta, n}\right\rangle V_{\alpha, i} k_{\beta, m}\right)
\end{aligned}
$$

(note that $G_{i j}$ is an estimator of $\partial V_{i} / \partial x_{j}$ ).

To study the dependency of physical and geometric errors on the shape parameters (elongation and planarity) of the Cluster tetrahedron, Chanteur (2000) assumed mutually uncorrelated spacecraft position vectors and measurements, and the errors to be isotropic. More precisely, for the covariance matrices he wrote

$$
\begin{aligned}
\left\langle\delta \boldsymbol{r}_{\mu} \delta \boldsymbol{r}_{\nu}^{\mathrm{t}}\right\rangle & =\delta_{\mu \nu}(\delta r)^{2} \mathbf{I} \quad \text { and } \\
\left\langle\delta \boldsymbol{V}_{\alpha} \delta \boldsymbol{V}_{\beta}^{\mathrm{t}}\right\rangle & =\delta_{\alpha \beta}(\delta V)^{2} \mathbf{I}
\end{aligned}
$$

where $\delta r$ and $\delta V$ denote the inaccuracies in position and field measurements, and $\delta_{\alpha \beta}$ as well as $\delta_{\mu \nu}$ are Kronecker delta symbols. These assumptions yield the following error formula

$\left\langle\delta G_{i j} \delta G_{m n}\right\rangle=\left[(\delta V)^{2} \delta_{i m}+\nabla V_{i} \cdot \nabla V_{m}(\delta r)^{2}\right] K_{j n}$,

see Eq. (11) in Chanteur (2000). Here the geometry of the spacecraft tetrahedron enters only through the reciprocal tensor $\mathbf{K}$. To illustrate the fundamental dependencies, we may reduce the complexity by considering the gradient estimator $\boldsymbol{G} \simeq \nabla g$ of a scalar variable $g$, so $G_{i j} \rightarrow G_{j}$, and we can write the covariance of the gradient vector as follows:

$\left\langle\delta[\nabla g] \delta[\nabla g]^{\mathrm{t}}\right\rangle=\left[(\delta g)^{2}+|\nabla g|^{2}(\delta r)^{2}\right] \mathbf{K}$.

The trace of this error formula gives the square magnitude error

$\left\langle|\delta \nabla g|^{2}\right\rangle=\left[(\delta g)^{2}+|\nabla g|^{2}(\delta r)^{2}\right] \operatorname{trace}(\mathbf{K})$

where the term

$\operatorname{trace}(\mathbf{K})=\sum_{\alpha=1}^{4}\left|\boldsymbol{k}_{\alpha}\right|^{2}$

is a function of the spacecraft array geometry, and can be understood as an amplification factor for the primary errors due to positional and measurement inaccuracies. For further details the reader is referred to Vogt and Paschmann (1998); Chanteur (2000); Vogt et al. (2008b).

Armed with the arsenal of error formulas for tetrahedral configurations, we now approach the three-point gradient estimation case. The correspondence of algebraic relations suggests the following replacement scheme

$$
\boldsymbol{k}_{\alpha} \rightarrow \boldsymbol{q}_{\alpha},
$$

$(\delta r)^{2} \mathbf{I} \rightarrow(\delta r)^{2}\left(\mathbf{I}-\hat{\boldsymbol{n}} \hat{\boldsymbol{n}}^{\mathrm{t}}\right)$,

$$
\begin{aligned}
& \sum_{\alpha=1}^{4} \rightarrow \sum_{\alpha=1}^{3}, \\
& \nabla V_{i} \rightarrow \nabla_{p} V_{i}
\end{aligned}
$$


in the four-point error formulas to find the corresponding expressions for the planar case. Assuming mutually uncorrelated and isotropic errors in spacecraft positions and measurements, the error formula for the covariance of the planar gradient vector of a scalar variable $g$ reads

$$
\left\langle\delta\left(\nabla_{p} g\right) \delta\left(\nabla_{p} g\right)^{\mathrm{t}}\right\rangle=\left[(\delta g)^{2}+\left|\nabla_{p} g\right|^{2}(\delta r)^{2}\right] \mathbf{Q},
$$

and for the square magnitude error we obtain

$$
\left\langle\left|\delta \nabla_{p} g\right|^{2}\right\rangle=\left[(\delta g)^{2}+\left|\nabla_{p} g\right|^{2}(\delta r)^{2}\right] \operatorname{trace}(\mathbf{Q}) .
$$

Hence in the three-point gradient estimation case, the array geometry amplifies the primary inaccuracies in spacecraft positions and measurements through the factor

$\operatorname{trace}(\mathbf{Q})=\sum_{\alpha=1}^{3}\left|\boldsymbol{q}_{\alpha}\right|^{2}$

In a similar way as trace $(\mathbf{K})$ can be written in terms of the shape parameters and an intrinsic scale of the four-spacecraft array (Chanteur, 2000), we may express the trace of the planar reciprocal tensor $\mathbf{Q}$ as a function of an intrinsic scale and the (planar) elongation $E_{p}$ of the three-spacecraft configuration. In analogy with the concept of planarity and elongation in the tetrahedral case (Robert et al., 1998b), we take the eigenvalues $R^{(1)} \geq R^{(2)} \geq R^{(3)}=0$ of the (singular) position tensor $\mathbf{R}=\sum_{\alpha=1}^{3} \boldsymbol{r}_{\alpha} \boldsymbol{r}_{\alpha}^{\mathrm{t}}$ of the three-spacecraft configuration to define

$$
E_{p}=1-\sqrt{R^{(2)} / R^{(1)}}
$$

and use the largest eigenvalue $R^{(1)}$ to identify an intrinsic scale

$L_{p}=\sqrt{R^{(1)}}$.

Note that Robert et al. (1998b) based their definitions on the eigenvalues of the volumetric tensor which differs from the position tensor by a constant factor, and that $R^{(3)}=0 \mathrm{im}$ plies $1-\sqrt{R^{(3)} / R^{(2)}}=1$, so the concept of planarity cannot be applied here. With $L_{p}$ and $E_{p}$ defined that way, the nonzero eigenvalues of the position tensor are $R^{(1)}=L_{p}^{2}$ and $R^{(2)}=L_{p}^{2}\left(1-E_{p}\right)^{2}$. Since in the three-spacecraft plane $\mathcal{P}$ the product $\mathbf{R Q}$ is the identity operation, the non-zero eigenvalues of $\mathbf{Q}$ are $Q^{(1)}=1 / R^{(1)}=L_{p}^{-2}$ and $Q^{(2)}=1 / R^{(2)}=$ $L_{p}^{-2}\left(1-E_{p}\right)^{-2}$. We finally write the trace of the planar reciprocal tensor in terms of the parameters $L_{p}$ and $E_{p}$ as follows:

$$
\operatorname{trace}(\mathbf{Q})=Q^{(1)}+Q^{(2)}=\frac{1}{L_{p}^{2}}\left(1+\frac{1}{\left(1-E_{p}\right)^{2}}\right)
$$

For the mean square error of the planar gradient magnitude we obtain

$$
\left\langle\left|\delta \nabla_{p} g\right|^{2}\right\rangle=\frac{\left(\delta_{p} g\right)^{2}}{L_{p}^{2}}\left(1+\frac{1}{\left(1-E_{p}\right)^{2}}\right)
$$

where the positional inaccuracies and the measurement errors have been combined into a single error variable:

$$
\delta_{p} g=\sqrt{(\delta g)^{2}+\left|\nabla_{p} g\right|^{2}(\delta r)^{2}} .
$$

\section{Appendix C}

\section{Accuracy of normal gradient estimation}

The component of a spatial gradient in the direction normal to the three-spacecraft plane cannot be estimated directly from measurements within that plane. Additional information in the form of dynamical or geometric constraints must be considered. Such conditions are never satisfied exactly.

In the following we focus on geometric constraints, and study how the estimation quality depends on small deviations from ideal geometries. If $\boldsymbol{G}$ denotes the true gradient and $\tilde{\boldsymbol{G}}$ the estimator, we evaluate the relative error in magnitude

$\frac{\delta G}{G}=\frac{\tilde{G}-G}{G}$

$(G=|\boldsymbol{G}|, \tilde{G}=|\tilde{\boldsymbol{G}}|)$ and the directional mismatch

$\sin \Delta=\left|\frac{\tilde{\boldsymbol{G}} \times \boldsymbol{G}}{\tilde{G} G}\right|$

where $\Delta \geq 0$ is the angle between the true gradient and the estimator. The geometric constraints of interest are $\hat{\boldsymbol{e}} \| \boldsymbol{G}$ and $\hat{\boldsymbol{e}} \perp \boldsymbol{G}$. The deviation from ideal geometry is quantified by the angle $\varepsilon$ between the error-free unit vector $\hat{\boldsymbol{e}}_{\circ}$ that fulfills the constraints exactly, and the unit vector $\hat{\boldsymbol{e}}$ that is actually used in the analysis:

$\cos \varepsilon=\cos \angle\left(\hat{\boldsymbol{e}}, \hat{\boldsymbol{e}}_{\circ}\right)=\hat{\boldsymbol{e}} \cdot \hat{\boldsymbol{e}}_{\circ}$.

The deviations from the ideal geometry considered here are assumed to be small: $\varepsilon \ll 1$.

True gradient and estimator are decomposed into planar and normal components:

$\begin{aligned} \boldsymbol{G} & =\boldsymbol{G}_{p}+\boldsymbol{G}_{n}=\boldsymbol{G}_{p}+G_{n} \hat{\boldsymbol{n}}, \\ \tilde{\boldsymbol{G}} & =\tilde{\boldsymbol{G}}_{p}+\tilde{\boldsymbol{G}}_{n}=\tilde{\boldsymbol{G}}_{p}+\tilde{G}_{n} \hat{\boldsymbol{n}} .\end{aligned}$

Since in this section we are concerned with the quality of the normal gradient estimate, we assume that the planar component of the gradient is known exactly:

$\tilde{\boldsymbol{G}}_{p}=\boldsymbol{G}_{p}$,

and write the normal component in the form

$\tilde{G}_{n}=G_{n}(1+v)$

where $v$ quantifies the relative deviation of the normal gradient estimator from its true value. It is straightforward to show 
that to lowest order in $v \ll 1$, the relative error in magnitude and the directional mismatch are given by

$$
\begin{aligned}
\frac{\delta G}{G} & \simeq \nu \frac{G_{n}^{2}}{G^{2}}, \\
\sin \Delta & \simeq\left|v \frac{G_{p} G_{n}}{G^{2}}\right| .
\end{aligned}
$$

Here and in the following the symbol " $\simeq$ " indicates that higher-order contributions in $v$ or $\varepsilon$ are neglected.

The coordinate system is chosen such that the true gradient points into the $z$-direction, and the normal unit vector $\hat{\boldsymbol{n}}$ is in the $(x, z)$-plane:

$$
\begin{aligned}
\boldsymbol{G} & =G \hat{z}, \\
\hat{\boldsymbol{n}} & =\sin \gamma \hat{\boldsymbol{x}}+\cos \gamma \hat{\boldsymbol{z}} .
\end{aligned}
$$

The angle between $\boldsymbol{G}$ and $\hat{\boldsymbol{n}}$ is $\gamma$. The components of the true gradient are

$$
\begin{aligned}
& G_{n}=\hat{\boldsymbol{n}} \cdot \boldsymbol{G}=G \cos \gamma, \\
& \boldsymbol{G}_{n}=G_{n} \hat{\boldsymbol{n}}=G \cos \gamma \sin \gamma \hat{\boldsymbol{x}}+G \cos ^{2} \gamma \hat{\boldsymbol{z}}, \\
& \boldsymbol{G}_{p}=\boldsymbol{G}-\boldsymbol{G}_{n}=-G \sin \gamma \cos \gamma \hat{\boldsymbol{x}}+G \sin ^{2} \gamma \hat{\boldsymbol{z}} .
\end{aligned}
$$

The geometric constraint $\hat{\boldsymbol{e}} \| \boldsymbol{G}$ is studied first which means that the normal gradient estimator is given by

$$
\tilde{G}_{n}=\frac{\left(\hat{\boldsymbol{e}} \cdot \boldsymbol{G}_{p}\right)(\hat{\boldsymbol{e}} \cdot \hat{\boldsymbol{n}})}{|\hat{\boldsymbol{e}} \times \hat{\boldsymbol{n}}|^{2}}
$$

We consider two types of $\hat{\boldsymbol{e}}$-variations around the exact vector $\hat{\boldsymbol{e}}_{\mathrm{o}}=\hat{z}$.

(a) The unit vector $\hat{\boldsymbol{e}}$ varies only in the $(x, z)$-plane:

$$
\hat{\boldsymbol{e}}=\sin \varepsilon \hat{\boldsymbol{x}}+\cos \varepsilon \hat{\boldsymbol{z}}
$$

i.e., in the plane spanned by $\boldsymbol{G}$ and $\hat{\boldsymbol{n}}$.

(b) The unit vector $\hat{\boldsymbol{e}}$ varies only in the $(y, z)$-plane:

$$
\hat{\boldsymbol{e}}=\sin \varepsilon \hat{\boldsymbol{y}}+\cos \varepsilon \hat{\boldsymbol{z}}
$$

i.e., in the direction perpendicular to the plane spanned by $\boldsymbol{G}$ and $\hat{\boldsymbol{n}}$.

We have to evaluate the scalar products $\hat{\boldsymbol{e}} \cdot \boldsymbol{G}_{p}$ and $\hat{\boldsymbol{e}} \cdot \hat{\boldsymbol{n}}$ as well as the square modulus of the cross product $|\hat{\boldsymbol{e}} \times \hat{\boldsymbol{n}}|^{2}$ in terms of the angles $\varepsilon$ and $\gamma$, then expand the resulting expressions in $\varepsilon \ll 1$ and neglect terms of higher than the leading order. Since the calculations are straightforward, details can be omitted, and only the end results are given.

For case (a) we obtain

$\tilde{G}_{n} \simeq G_{n}\left(1+\frac{\varepsilon}{\sin \gamma \cos \gamma}\right)=G_{n}(1+v)$ thus $v=\varepsilon \cdot(\sin \gamma \cos \gamma)^{-1}$. With $G_{n} / G=\cos \gamma$ and $G_{p} / G=$ $\sin \gamma$ we get

$$
\begin{gathered}
\frac{\delta G}{G} \simeq \nu \frac{G_{n}^{2}}{G^{2}}=\varepsilon \frac{\cos \gamma}{\sin \gamma}, \\
\sin \Delta \simeq\left|\nu \frac{G_{p} G_{n}}{G^{2}}\right|=\varepsilon .
\end{gathered}
$$

The magnitude estimate is most susceptible to errors in $\hat{\boldsymbol{e}}$ if $\gamma$ is small, i.e., if the true gradient is close to the normal direction. To lowest order in $\varepsilon$, the magnitude estimate is rather robust if the gradient is close to the three-spacecraft plane, and the directional mismatch does not depend on $\gamma$ at all.

Case (b) yields

$\tilde{G}_{n} \simeq G_{n}\left(1-\varepsilon^{2}\left[\frac{1}{2}+\frac{1}{\sin ^{2} \gamma}\right]\right)$

which gives $v=-\varepsilon^{2}\left[1 / 2+1 / \sin ^{2} \gamma\right]$, and

$$
\begin{aligned}
\frac{\delta G}{G} & \simeq-\varepsilon^{2} \cos ^{2} \gamma\left[\frac{1}{2}+\frac{1}{\sin ^{2} \gamma}\right], \\
\sin \Delta & \simeq \varepsilon^{2} \cos \gamma\left[\frac{\sin \gamma}{2}+\frac{1}{\sin \gamma}\right] .
\end{aligned}
$$

The expansions in $\varepsilon$ both lack the linear order which means the corresponding estimates are more robust with respect to uncertainties of the vector $\hat{\boldsymbol{e}}$ in the direction perpendicular to the plane spanned by $\boldsymbol{G}$ and $\hat{\boldsymbol{n}}$.

Now we study the second type of geometric constraint, namely, $\hat{\boldsymbol{e}} \perp \boldsymbol{G}$ where the normal gradient estimator is given by

$\tilde{G}_{n}=-\frac{\hat{\boldsymbol{e}} \cdot \boldsymbol{G}_{p}}{\hat{\boldsymbol{e}} \cdot \hat{\boldsymbol{n}}}$.

As before we distinguish two types of $\hat{\boldsymbol{e}}$-variations around the exact vector which now resides somewhere in the $(x, y)$ plane:

$\hat{\boldsymbol{e}}_{\circ}=\cos \phi \hat{\boldsymbol{x}}+\sin \phi \hat{\boldsymbol{y}}$.

Here $\phi$ is the angle between $\hat{\boldsymbol{e}}_{\circ}$ and the $x$-axis.

(c) The unit vector $\hat{\boldsymbol{e}}$ varies only in the $(x, y)$-plane:

$$
\hat{\boldsymbol{e}}=\cos (\phi+\varepsilon) \hat{\boldsymbol{x}}+\sin (\phi+\varepsilon) \hat{\boldsymbol{y}},
$$

i.e., in the plane perpendicular to $\boldsymbol{G}$.

(d) The unit vector $\hat{\boldsymbol{e}}$ varies only in the $z$-direction:

$$
\hat{\boldsymbol{e}}=\cos \varepsilon \cos \phi \hat{\boldsymbol{x}}+\cos \varepsilon \sin \phi \hat{\boldsymbol{y}}+\sin \varepsilon \hat{\boldsymbol{z}} .
$$

i.e., in the direction parallel to $\boldsymbol{G}$. 
For the type of variation given in (c) we obtain

$\tilde{G}_{n}=G_{n}$

thus $v=0$, and there is no error in gradient magnitude or direction associated with this kind of variation in $\hat{\boldsymbol{e}}$. This is not surprising as the geometric constraint $\hat{\boldsymbol{e}} \perp \boldsymbol{G}$ is preserved if $\hat{\boldsymbol{e}}$ is varied only in the $(x, y)$-plane, i.e., in the plane perpendicular to the true gradient $\boldsymbol{G}$.

The configuration in (d) yields

$$
\tilde{G}_{n} \simeq G_{n}\left(1-\frac{\varepsilon}{\sin \gamma \cos \gamma \cos \phi}\right)
$$

thus $v=-\varepsilon \cdot(\sin \gamma \cos \gamma \cos \phi)^{-1}$, and

$$
\begin{aligned}
\frac{\delta G}{G} & \simeq-\varepsilon \frac{\cos \gamma}{\sin \gamma \cos \phi}, \\
\sin \Delta & \simeq \varepsilon \frac{1}{\cos \phi} .
\end{aligned}
$$

As in case (a), the magnitude estimate is affected heavily by uncertainties in $\hat{\boldsymbol{e}}$ if $\gamma$ is small, i.e., if the gradient is perpendicular to the three-spacecraft plane, whereas to lowest order in $\varepsilon$, the directional mismatch is not sensitive to the value of $\gamma$. A new aspect is the orientation of $\hat{\boldsymbol{e}}$ with respect to the normal direction $\hat{\boldsymbol{n}}$ : uncertainties in $\hat{\boldsymbol{e}}$ are amplified strongly if $\cos \phi \approx 0$, i.e., if $\hat{\boldsymbol{e}} \perp \hat{\boldsymbol{n}}$ or, equivalently, if $\hat{\boldsymbol{e}}$ is in the threespacecraft plane.

Finally, we look at the dynamical constraint which is based on the assumption that the time derivative $\mathrm{d} / \mathrm{d} t$ vanishes in a frame of reference moving with the velocity $\boldsymbol{U}$. A known residual time derivative $\mathrm{d} / \mathrm{d} t \neq 0$ could easily be taken into account in Eq. (36) by replacing $\partial / \partial t \rightarrow \partial / \partial t-\mathrm{d} / \mathrm{d} t$. Therefore, to simplify the error analysis, the uncertainty in $\mathrm{d} g / \mathrm{d} t$ can be combined with the error of $\partial g / \partial t$ if we interpret $\delta[\partial g / \partial t]$ as the deviation of the estimated $\partial g / \partial t$ from the true $(\partial g / \partial t-\mathrm{d} g / \mathrm{d} t)$. The resulting uncertainty in the normal derivative estimate can be written as

$\delta\left[\frac{\partial g}{\partial n}\right]=-\frac{1}{U_{n}} \delta\left[\frac{\partial g}{\partial t}\right]$.

The error is amplified through the term $1 / U_{n}$ which assumes large values if $U_{n}$ is small.

Acknowledgements. This work was initiated at the 6th COSPAR Capacity Building Workshop (STIINTE) in Sinaia, Romania, in June 2007. J. V. thanks the co-organizers, the workshop participants, and the sponsors of this memorable event. The work of A. A. was financially supported by the Konrad-Adenauer-Stiftung. O. M. acknowledges funding through the PECS contract ECSTRA, C98048, and the kind hospitality of Max-Planck-Institut für extraterrestrische Physik, Garching. The authors acknowledge the teams of the FGM and the CIS instruments on ESA's Cluster spacecraft, the CIS data repository in Toulouse, and the Cluster Active Archive (CAA).

Topical Editor I. A. Daglis thanks J. de Keyser and another anonymous referee for their help in evaluating this paper.

\section{References}

Balikhin, M. A. and Gedalin, M. E.: Comparative analysis of different methods for distinguishing temporal and spatial variations, in: ESA WPP-047: Proc. of Start Conf., Aussois, France, pp. 183-187, 1993.

Balogh, A., Carr, C. M., Acuña, M. H., Dunlop, M. W., Beek, T. J., Brown, P., Fornacon, K.-H., Georgescu, E., Glassmeier, K.H., Harris, J., Musmann, G., Oddy, T., and Schwingenschuh, K.: The Cluster Magnetic Field Investigation: overview of in-flight performance and initial results, Ann. Geophys., 19, 1207-1217, 2001, http://www.ann-geophys.net/19/1207/2001/.

Bargatze, L. F., McPherron, R. L., Minamora, J., and Weimer, D.: A new interpretation of Weimer et al.'s solar wind propagation delay technique, J. Geophys. Res., 110, A07105, doi:10.1029/ 2004JA010902, 2005.

Bargatze, L. F., McPherron, R. L., Minamora, J., and Weimer, D.: Reply to comment by Haaland et al. on "A new interpretation of Weimer et al.'s solar wind propagation delay technique", J. Geophys. Res., 111, A06103, doi:10.1029/2005JA011557, 2006.

Chanteur, G.: Spatial Interpolation for Four Spacecraft: Theory, in: Analysis Methods for Multi-Spacecraft Data, edited by: Paschmann, G. and Daly, P., pp. 371-393, ISSI/ESA, 1998.

Chanteur, G.: Accuracy of field gradient estimations by Cluster: Explanation of its dependency upon elongation and planarity of the tetrahedron, pp. 265-268, ESA SP-449, 2000.

Chanteur, G. and Harvey, C. C.: Spatial Interpolation for Four Spacecraft: Application to Magnetic Gradients, in: Analysis Methods for Multi-Spacecraft Data, edited by: Paschmann, G. and Daly, P., pp. 349-369, ISSI/ESA, 1998.

Constantinescu, O. D., Glassmeier, K.-H., Dcrau, P. M. E., Fränz, M., and Fornaon, K.-H.: Low frequency wave sources in the outer magnetosphere, magnetosheath, and near Earth solar wind, Ann. Geophys., 25, 2217-2228, 2007, http://www.ann-geophys.net/25/2217/2007/.

De Keyser, J.: Least-squares multi-spacecraft gradient calculation with automatic error estimation, Ann. Geophys., 26, 3295-3316, 2008, http://www.ann-geophys.net/26/3295/2008/.

De Keyser, J., Roth, M., Dunlop, M. W., Rme, H., Owen, C. J., and Paschmann, G.: Empirical reconstruction and long-duration tracking of the magnetospheric boundary in single- and multispacecraft contexts, Ann. Geophys., 23, 1355-1369, 2005, http://www.ann-geophys.net/23/1355/2005/.

De Keyser, J., Darrouzet, F., Dunlop, M. W., and Décréau, P. M. E.: Least-squares gradient calculation from multi-point observations of scalar and vector fields: methodology and applications with Cluster in the plasmasphere, Ann. Geophys., 25, 971-987, 2007, http://www.ann-geophys.net/25/971/2007/.

Dudok de Wit, T., Krasnoselskikh, V. V., Bale, S. D., Dunlop, M. W., Lühr, H., Schwartz, S. J., and Woolliscroft, L. J. C.: Determination of dispersion relations in quasi-stationary plasma turbulence using dual satellite data, Geophys. Res. Lett., 22, $2653-$ 2656, doi:10.1029/95GL02543, 1995.

Dunlop, M. W. and Eastwood, J. P.: The Curlometer and Other Gradient Based Methods, pp. 17-25, ISSI SR-008, 2008.

Dunlop, M. W. and Woodward, T. I.: Multi-Spacecraft Discontinuity Analysis: Orientation and Motion, in: Analysis Methods for Multi-Spacecraft Data, edited by: Paschmann, G. and Daly, P., pp. 271-305, ISSI/ESA, 1998.

Dunlop, M. W., Southwood, D. J., Glassmeier, K.-H., and 
Neubauer, F. M.: Analysis of multipoint magnetometer data, Adv. Space Res., 8, 273-277, 1988.

Dunlop, M. W., Balogh, A., Glassmeier, K.-H., and Robert, P.: Four-point Cluster application of magnetic field analysis tools: The Curlometer, J. Geophys. Res., 107, 1384, doi:10.1029/ 2001JA005088, 2002.

Haaland, S., Paschmann, G., and Sonnerup, B. U. Ö.: Comment on "A new interpretation of Weimer et al.'s solar wind propagation delay technique" by Bargatze et al., J. Geophys. Res., 111, A06102, doi:10.1029/2005JA011376, 2006.

Hamrin, M., Marghitu, O., Rönnmark, K., Klecker, B., André, M., Buchert, S., Kistler, L. M., McFadden, J., Rème, H., and Vaivads, A.: Observations of concentrated generator regions in the nightside magnetosphere by Cluster/FAST conjunctions, Ann. Geophys., 24, 637-649, 2006,

http://www.ann-geophys.net/24/637/2006/.

Hamrin, M., Rönnmark, K., Börlin, N., Vedin, J., and Vaivads, A.: GALS - Gradient Analysis by Least Squares, Ann. Geophys., 26, 3491-3499, 2008, http://www.ann-geophys.net/26/3491/2008/.

Harvey, C. C.: Spatial Gradients and the Volumetric Tensor, pp. 307-322, ISSI SR-001, 1998.

Horbury, T. S. and Osman, K. T.: Multi-Spacecraft Turbulence Analysis Methods, pp. 55-64, ISSI SR-008, 2008.

Knetter, T., Neubauer, F. M., Horbury, T., and Balogh, A.: Fourpoint discontinuity observations using Cluster magnetic field data: A statistical survey, J. Geophys. Res., 109, A06102, doi: 10.1029/2003JA010099, 2004.

Marghitu, O., Hamrin, M., Klecker, B., Vaivads, A., McFadden, J., Buchert, S., Kistler, L. M., Dandouras, I., André, M., and Rème, H.: Experimental investigation of auroral generator regions with conjugate Cluster and FAST data, Ann. Geophys., 24, 619-635, 2006, http://www.ann-geophys.net/24/619/2006/.

Motschmann, U., Glassmeier, K.-H., and Pinçon, J. L.: MultiSpacecraft Filtering: Plasma Mode Recognition, in: Analysis Methods for Multi-Spacecraft Data, edited by: Paschmann, G. and Daly, P., pp. 65-78, ISSI/ESA, 1998.

Neubauer, F. M. and Glassmeier, K.-H.: Use of an array of satellites as a wave telescope, J. Geophys. Res., 95, 19115-19122, 1990.

Paschmann, G. and Daly, P. W.: Analysis Methods for MultiSpacecraft Data, no. SR-001 in ISSI Scientific Reports, ESA Publ. Div., Noordwijk, Netherlands, 1998.

Paschmann, G. and Daly, P. W.: Multi-Spacecraft Analysis Methods Revisited, no. SR-008 in ISSI Scientific Reports, ESA Publ. Div., Noordwijk, Netherlands, 2008.

Pinçon, J.-L. and Glassmeier, K.-H.: Multi-Spacecraft Methods of Wave Field Characterisation, pp. 47-54, ISSI SR-008, 2008.

Pinçon, J. L. and Lefeuvre, F.: Local characterization of homogeneous turbulence in a space plasma from simultaneous measurements of field components at several points in space, J. Geophys. Res., 96, 1789-1802, 1991.

Pinçon, J. L. and Lefeuvre, F.: The application of the generalized Capon method to the analysis of a turbulent field in space plasma - Experimental constraints, J. Atmos. Terr. Phys., 54, 12371247, 1992.

Pinçon, J. L. and Motschmann, U.: Multi-Spacecraft Filtering: General Framework, in: Analysis Methods for Multi-Spacecraft Data, edited by: Paschmann, G. and Daly, P. , pp. 65-78, ISSI/ESA, 1998.
Rème, H., Aoustin, C., Bosqued, J. M., Dandouras, I., Lavraud, B., Sauvaud, J. A., Barthe, A., Bouyssou, J., Camus, Th., CoeurJoly, O., Cros, A., Cuvilo, J., Ducay, F., Garbarowitz, Y., Medale, J. L., Penou, E., Perrier, H., Romefort, D., Rouzaud, J., Vallat, C., Alcaydé, D., Jacquey, C., Mazelle, C., d’Uston, C., Möbius, E., Kistler, L. M., Crocker, K., Granoff, M., Mouikis, C., Popecki, M., Vosbury, M., Klecker, B., Hovestadt, D., Kucharek, H., Kuenneth, E., Paschmann, G., Scholer, M., Sckopke, N., Seidenschwang, E., Carlson, C. W., Curtis, D. W., Ingraham, C., Lin, R. P., McFadden, J. P., Parks, G. K., Phan, T., Formisano, V., Amata, E., Bavassano-Cattaneo, M. B., Baldetti, P., Bruno, R., Chionchio, G., Di Lellis, A., Marcucci, M. F., Pallocchia, G., Korth, A., Daly, P. W., Graeve, B., Rosenbauer, H., Vasyliunas, V., McCarthy, M., Wilber, M., Eliasson, L., Lundin, R., Olsen, S., Shelley, E. G., Fuselier, S., Ghielmetti, A. G., Lennartsson, W., Escoubet, C. P., Balsiger, H., Friedel, R., Cao, J.-B., Kovrazhkin, R. A., Papamastorakis, I., Pellat, R., Scudder, J., and Sonnerup, B.: First multispacecraft ion measurements in and near the Earth's magnetosphere with the identical Cluster ion spectrometry (CIS) experiment, Ann. Geophys., 19, 1303-1354, 2001, http://www.ann-geophys.net/19/1303/2001/.

Robert, P., Dunlop, M. D., Roux, A., and Chanteur, G.: Accuracy of Current Density Estimation, pp. 395-418, ISSI SR-001, 1998a.

Robert, P., Roux, A., Harvey, C. C., Dunlop, M. W., Daly, P. W., and Glassmeier, K.-H.: Tetrahedron Geometric Factors, pp. 323-348, ISSI SR-001, 1998b.

Runov, A., Sergeev, V. A., Baumjohann, W., Nakamura, R., Apatenkov, S., Asano, Y., Volwerk, M., Vrös, Z., Zhang, T. L., Petrukovich, A., Balogh, A., Sauvaud, J.-A., Klecker, B., and Rème, H.: Electric current and magnetic field geometry in flapping magnetotail current sheets, Ann. Geophys., 23, 1391-1403, 2005, http://www.ann-geophys.net/23/1391/2005/.

Runov, A., Sergeev, V. A., Nakamura, R., Baumjohann, W., Apatenkov, S., Asano, Y., Takada, T., Volwerk, M., Vörös, Z., Zhang, T. L., Sauvaud, J.-A., Rème, H., and Balogh, A.: Local structure of the magnetotail current sheet: 2001 Cluster observations, Ann. Geophys., 24, 247-262, 2006, http://www.ann-geophys.net/24/247/2006/.

Samson, J. C., Greenwald, R. A., Ruohoniemi, J. M., Frey, A., and Baker, K. B.: Goose Bay radar observations of earth-reflected, atmospheric gravity waves in the high-latitude ionosphere, J. Geophys. Res., 95, 7693-7709, 1990.

Santolík, O., Parrot, M., and Lefeuvre, F.: Singular value decomposition methods for wave propagation analysis, Radio Sci., 38, 10-1, doi:10.1029/2000RS002523, 2003.

Siscoe, G. L., Davis, Jr., L., Coleman, Jr., P. J., Smith, E. J., and Jones, D. E.: Power Spectra and Discontinuities of the Interplanetary Magnetic Field: Mariner 4, J. Geophys. Res., 73, 61-82, doi:10.1029/JA073i001p00061, 1968.

Sonnerup, B. U. O. and Cahill Jr., L. J.: Magnetopause Structure and Attitude from Explorer 12 Observations, J. Geophys. Res., 72, 171-183, 1967.

Sonnerup, B. U. Ö. and Scheible, M.: Minimum and Maximum Variance Analysis, in: Analysis Methods for Multi-Spacecraft Data, edited by: Paschmann, G. and Daly, P., pp. 185-220, ISSI/ESA, 1998.

Sonnerup, B. U. Ö., Haaland, S., and Paschmann, G.: Discontinuity Orientation, Motion, and Thickness, pp. 1-15, ISSI SR-008, 2008. 
Vallat, C., Dandouras, I., Dunlop, M., Balogh, A., Lucek, E., Parks, G. K., Wilber, M., Roelof, E. C., Chanteur, G., and Rème, H.: First current density measurements in the ring current region using simultaneous multi-spacecraft CLUSTER-FGM data, Ann. Geophys., 23, 1849-1865, 2005,

http://www.ann-geophys.net/23/1849/2005/.

Vogt, J. and Paschmann, G.: Accuracy of Plasma Moment Derivatives, pp. 419-447, ISSI SR-001, 1998.

Vogt, J., Narita, Y., and Constantinescu, O. D.: The wave surveyor technique for fast plasma wave detection in multi-spacecraft data, Ann. Geophys., 26, 1699-1710, 2008a, http://www.ann-geophys.net/26/1699/2008/.
Vogt, J., Paschmann, G., and Chanteur, G.: Reciprocal Vectors, pp. 33-46, ISSI SR-008, 2008b.

Walker, S. N., Sahraoui, F., Balikhin, M. A., Belmont, G., Pinon, J. L., Rezeau, L., Alleyne, H., Cornilleau-Wehrlin, N., and André, M.: A comparison of wave mode identification techniques, Ann. Geophys., 22, 3021-3032, 2004, http://www.ann-geophys.net/22/3021/2004/.

Weimer, D. R. and King, J. H.: Improved calculations of interplanetary magnetic field phase front angles and propagation time delays, J. Geophys. Res., 113, A01105, doi:10.1029/ 2007JA012452, 2008. 